\title{
Two-twistor particle models and free massive higher spin fields
}

\author{
J.A. de Azcárraga, ${ }^{a}$ S. Fedoruk, ${ }^{b, c, 1}$ J.M. Izquierdo ${ }^{d}$ and J. Lukierski ${ }^{e}$ \\ ${ }^{a}$ Department of Theoretical Physics and IFIC (CSIC-UVEG), Valencia University, \\ 46100-Burjassot (Valencia), Spain \\ ${ }^{b}$ Bogolubov Laboratory of Theoretical Physics, Joint Institute for Nuclear Research, \\ Joliot-Curie 6, 141980 Dubna, Moscow region, Russia \\ ${ }^{c}$ Department of Theoretical Physics, Tomsk State Pedagogical University, \\ Tomsk, 634061, Russia \\ ${ }^{d}$ Department of Theoretical Physics, Valladolid University, \\ 47011-Valladolid, Spain \\ ${ }^{e}$ Institute of Theoretical Physics, Wroctaw University, \\ pl. Maxa Borna 9, 50-204 Wroctaw, Poland \\ E-mail: j.a.de.azcarraga@ific.uv.es, fedoruk@theor.jinr.ru, \\ izquierd@fta.uva.es, jerzy.lukierski@ift.uni.wroc.pl
}

Abstract: We present $D=3$ and $D=4$ world-line models for massive particles moving in a new type of enlarged spacetime, with $D-1$ additional vector coordinates, which after quantization lead to towers of massive higher spin (HS) free fields. Two classically equivalent formulations are presented: one with a hybrid spacetime/bispinor variables and a second described by a free two-twistor dynamics with constraints. After first quantization in the $D=3$ and $D=4$ cases, the wave functions satisfying a massive version of Vasiliev's free unfolded equations are given as functions on the $\operatorname{SL}(2, \mathbb{R})$ and $\operatorname{SL}(2, \mathbb{C})$ group manifolds respectively, which describe arbitrary on-shell momenta and spin degrees of freedom. Further we comment on the $D=6$ case, and possible supersymmetric extensions are mentioned as well. Finally, the description of interactions and the AdS/CFT duality are briefly considered for massive HS fields.

Keywords: Field Theories in Lower Dimensions, Higher Spin Symmetry, Extended Supersymmetry, Space-Time Symmetries

ARXIV EPRINT: 1409.7169

\footnotetext{
${ }^{1}$ On leave of absence from V.N. Karazin Kharkov National University, Ukraine.
} 


\section{Contents}

$2 \quad D=3$ bispinorial particle models and HS massive fields from their quantization

2.1 Summary of $D=3$ two-twistor kinematics 5

2.2 $D=3$ bispinorial generalization of the Shirafuji model $\quad 7$

$2.3 D=3$ bitwistorial description $\quad 9$

$3 \quad D=4$ bispinorial models and HS massive fields $\quad 14$

$\begin{array}{lll}3.1 & \text { Summary of } D=4 \text { two-twistor kinematics } & 14\end{array}$

$\begin{array}{ll}3.2 D=4 \text { bispinorial generalization of Shirafuji model } & 15\end{array}$

$\begin{array}{lll}3.3 D & D=4 \text { bitwistorial description of HS massive multiplets } & 18\end{array}$

$3.4 D=4$ bitwistor wave function of HS massive multiplet 21

4 Outlook 26

$\begin{array}{ll}\text { A Notation } & \mathbf{3 0}\end{array}$

B From $D=3$ spinorial to $D=4$ vectorial particle model 32

\section{Introduction}

The development of higher spin (HS) theory was predominantly associated with massless (conformal) HS fields. One of the important methods for the description of HS fields consists of introducing master fields on an enlarged spacetime, which then lead to spacetime fields with all possible values of helicity (when the mass $m=0$ ) or spin (when $m \neq 0$ ). In particular, a collection of $D=4$ massless HS fields with arbitrary helicities was described by quantizing particles propagating in tensorial spacetime $x_{M}=\left(x_{\mu} \sim x_{\alpha \dot{\beta}}, y_{\mu \nu} \sim\left(y_{\alpha \beta}, \bar{y}_{\dot{\alpha} \dot{\beta}}\right)\right)$ extended by commuting Weyl spinor coordinates $y_{\alpha}, y_{\dot{\alpha}}, \alpha, \dot{\alpha}=1,2$ (see e.g. [1-6]; for the spinorial notation, see appendix A). It is easy to show that an equivalent particle model can be formulated in twistor space [7, 8], with tensorial spacetime coordinates eliminated by generalized Penrose incidence relations [2-6].

In this paper we consider the description of free massive HS fields, obtained by quantization of a new particle world-line model in $D=4$ generalized spacetime $X_{\mathcal{M}}=\left(x_{\mu}, y_{\mu}^{r}\right)$ $(r=1,2,3)$ extended by the pair of commuting Weyl spinors $y_{\alpha}^{i}, \bar{y}_{\dot{\alpha} i}(i=1,2)$. We recall that generalized spacetime with one auxiliary fourvector $y_{\mu}$ had been employed for a bilocal description of infinite massless HS multiplets already in the seventies [9] (see further [10]). We also add that recently, in the context of AdS/CFT duality, a similar bilocal description 
has been obtained from first quantization of a world-line biparticle model [11, 12]. In our approach, we shall supplement spacetime with three auxiliary vectors (in $D=4$ providing 12 degrees of freedom), but due to the phase space constraints which follow from our particle model most of these degrees are non-dynamical.

Let us recall the considerations in [2-6]. The most general $D=4$ model in $D=4$ tensorial spacetime describing free HS multiplets is provided by the following action

$$
S=\int d \tau\left(\pi_{\alpha} \bar{\pi}_{\dot{\beta}} \dot{x}^{\alpha \dot{\beta}}+a \pi_{\alpha} \pi_{\beta} \dot{y}^{\alpha \beta}+\bar{a} \bar{\pi}_{\dot{\alpha}} \bar{\pi}_{\dot{\beta}} \dot{\bar{y}}^{\dot{\alpha} \dot{\beta}}+b \pi_{\alpha} \dot{y}^{\alpha}+\bar{b} \bar{\pi}_{\dot{\alpha}} \dot{\bar{y}}^{\dot{\alpha}}\right),
$$

where $a, b$ are complex parameters, $\bar{\pi}_{\dot{\alpha}} \equiv\left(\pi_{\alpha}\right)^{*}$, etc. The model (1.1) with $b=0$ was considered in [3], and the last two terms $(b \neq 0)$ were first introduced in [4]. The advantage of having $b \neq 0$ is the much simpler structure of the constraints in phase space and the easier quantization procedure. It turns out that for $a \neq 0$ and/or $b \neq 0$ the hybrid action (1.1) depending on tensorial spacetime and spinorial coordinates can be rewritten (modulo boundary terms) as the one-twistor free particle model $[7,8]$

$$
\begin{aligned}
S & =-\frac{1}{2} \int d \tau\left(\bar{Z}_{A} \dot{Z}^{A}+\text { h.c. }\right)=-\frac{1}{2} \int d \tau\left(\omega^{\alpha} \dot{\pi}_{\alpha}-\bar{\pi}_{\dot{\alpha}} \dot{\bar{\omega}}^{\dot{\alpha}}+\text { h.c. }\right) \\
& =-\int d \tau\left(\omega^{\alpha} \dot{\pi}_{\alpha}-\bar{\pi}_{\dot{\alpha}} \dot{\bar{\omega}}^{\dot{\alpha}}\right)+\text { boundary term }
\end{aligned}
$$

and the $D=4$ twistor $Z^{A}, A=1, \ldots, 4$ (conformal basic spinor) is described by a pair of Weyl spinors

$$
Z^{A}=\left(\begin{array}{c}
\pi_{\alpha} \\
\bar{\omega}^{\dot{\alpha}}
\end{array}\right), \quad\left(Z^{A}\right)^{\dagger}=\left(\bar{\pi}_{\dot{\alpha}}, \omega^{\alpha}\right)
$$

where the conformally invariant scalar product

$$
\bar{Z}_{A} Z^{A} \equiv\left(Z^{A}\right)^{\dagger} g_{A B} Z^{B}=\omega^{\alpha} \pi_{\alpha}-\bar{\pi}_{\dot{\alpha}} \bar{\omega}^{\dot{\alpha}}
$$

is obtained by the particular choice of the anti-hermitian antisymmetric $\mathrm{U}(2,2)$ metric $^{1}$

$$
g_{A B}=\left(\begin{array}{cc}
0 & -\delta^{\dot{\alpha}} \dot{\beta} \\
\delta_{\alpha}{ }^{\beta} & 0
\end{array}\right) .
$$

The passage from the hybrid spacetime/spinor description (1.1) to the twistorial one (1.2) is achieved by a modified Penrose incidence relation. For the actions (1.1) and (1.2) a suitably chosen incidence relation is:

$$
\begin{aligned}
& \omega^{\alpha}=x^{\alpha \dot{\beta}} \bar{\pi}_{\dot{\beta}}+2 a y^{\alpha \beta} \pi_{\beta}+b y^{\alpha}, \\
& \bar{\omega}^{\dot{\alpha}}=\pi_{\beta} x^{\beta \dot{\alpha}}+2 \bar{a} \bar{y}^{\dot{\alpha} \dot{\beta}} \bar{\pi}_{\dot{\beta}}+\bar{b} \bar{y}^{\dot{\alpha}} .
\end{aligned}
$$

\footnotetext{
${ }^{1}$ The choice $(1.5)$ is used in $[13,14]$ and has been adjusted in such a way that it remains valid also for real $D=3$ twistors, which are fundamental $\operatorname{Sp}(4 ; \mathbb{R})$ spinors (see section 2.1 ). In $D=4$ this choice of the $\mathrm{SU}(2,2)$ metric leads to purely imaginary twistor lengths (see (1.4)). Note that the conformal groups $\mathrm{SO}(2, \nu+2)(\nu=1,2,4)$ in spacetime dimensions $D=\nu+2$ are isomorphic to the $U_{\alpha}(4 ; \mathbb{K})$ groups, where $\mathbb{K}=\mathbb{R}, \mathbb{C}, \mathbb{H}$ are the corresponding division algebras and $U_{\alpha}(2 n ; \mathbb{K})$ are the antiunitary $\mathbb{K}$-valued matrix groups preserving the anti-hermitian bilinear form. We have $U_{\alpha}(2 n ; \mathbb{R}) \simeq \mathrm{Sp}(2 n ; \mathbb{R}), U_{\alpha}(2 n ; \mathbb{C}) \simeq \mathrm{U}(n, n)$ and $U_{\alpha}(2 n ; \mathbb{H}) \simeq O(2 n ; \mathbb{H}) \simeq O^{*}(4 n ; \mathbb{C})$ (see e.g. [15]).
} 
After inserting eqs. (1.6) into (1.1), the free twistorial particle action (1.2) follows modulo boundary terms. Besides, since $x^{\alpha \dot{\beta}}$ in the action (1.1) has to be hermitian for $x^{\mu}$ to be real, inserting (1.6) in eq. (1.4) we see that

$$
\bar{Z}_{A} Z^{A}=\left(2 a \pi_{\alpha} \pi_{\beta} y^{\alpha \beta}-\text { h.c. }\right)+\left(b \pi_{\alpha} y^{\alpha}-\text { h.c. }\right) .
$$

Using the realization of the Poincaré algebra in terms of the twistor coordinates $Z^{A}, \bar{Z}_{A}$ (see $[7,8,16]$ ), and using the canonical Poisson brackets (PB) following from (1.2), it follows that in the $D=4$ massless case the helicity $h$ is given by

$$
h=\frac{i}{2} \bar{Z}_{A} Z^{A} .
$$

When $a=b=0$ we obtain the Shirafuji model [8] with twistor coordinates restricted, due to (1.7), by the zero helicity constraint $\bar{Z}_{A} Z^{A}=0$. In the twistor formulation of the Shirafuji model (1.2), this helicity constraint has to be added by a Lagrange multiplier. We add that the zero value of helicity can be shifted after quantization $(h \rightarrow \hat{h})$ to a non-zero one by using various orderings for the quantized twistors in the helicity operator $\hat{h}[17,18]$. If $a \neq 0$ and/or $b \neq 0$ the value of $h$ (see (1.7)) is not kinematically restricted in the twistor framework and the action describes an infinite massless multiplet with all helicities (see e.g. [3]).

The HS theory formulated on generalized spacetimes with supplementary spinorial coordinates has been employed by Vasiliev and his collaborators for the description of interacting massless HS fields since early 90's [4, 18-26]. The dynamics of free HS master fields, derived in [3-6] from a first-quantization of the particle model (1.1), corresponds in Vasiliev theory to the simplest "free" choice of general unfolded equations ${ }^{2}$ which, in the general case, provide the description of interacting HS gauge fields (for recent reviews se $[30,31]$ ). The unfolded equations for massive HS fields in Minkowski or AdS spacetimes have also been treated in Vasiliev framework [32-38], although without sufficiently conclusive or general results. In this paper we propose a new type of unfolded equations for free massive HS fields. The novelty of our approach is the new extension of $D=3$ and $D=4$ spacetime by $D-1$ auxiliary vectors which is dual to extended momentum space with orthogonal Lorentz frame constraints. The mass and the spin are introduced geometrically and, generalizing the method for the massless HS case [2-6], we formulate a new particle model in two-twistor space with suitable constraints.

In this paper we describe $D=3$ and $D=4$ HS particle models which, after first quantization, lead to free massive HS fields with arbitrary values of spin. The application of the ideas presented in [3-6] to the massive case requires the doubling of spinor indices in the hybrid (eq. (1.1)) particle actions (see e.g. [39-43]) and the enlargement of (1.2) to the free two-twistor action (see e.g. [44-52]). In our study we provide the generalizations of the actions (1.1) and (1.2) by incorporating the mass-shell constraints and by introducing a

\footnotetext{
${ }^{2}$ The unfolding technique consists in replacing the higher order equations for the dynamical variables of the original system by an equivalent first order formulation obtained by adding suitable auxiliary variables. In unfolded HS dynamics one introduces infinitely many nondynamical auxiliary fields; the name "unfolding" was introduced in [27]. It has been known for a long time that HS equations in spacetime require higher order derivatives $[28,29]$.
} 
suitable form of the incidence relations linking the two-twistorial and generalized spacetime coordinates. In this way, we obtain HS particle models with the right number of physical phase space degrees of freedom, namely six in $D=3$ (abelian spins) and twelve degrees of freedom in $D=4$ (SU(2)-spins). It will follow that describing massive HS fields by an extension of the 'hybrid' (eq. (1.1)) and purely twistorial (eq. (1.2)) actions produces equivalent models with the same number of degrees of freedom.

The plan of the paper is as follows. In section 2 we study $D=3$ massive HS models. After some kinematic results about $D=3$ two-twistor space we describe our $D=3$ counterpart of the model (1.1). It is shown that the standard two-twistor Shirafuji model without additional coordinates only provides spinless massive $D=3$ particles (see also [43]). To modify this result in order to obtain $D=3$ massive particles with arbitrary spin, we introduce a spinorial action with a pair of additional three-vector coordinates and we impose suitable mass constraints. Further, we describe the model in phase space and show that after solving the first class constraints providing the unfolded equations, we obtain a wave function on the three-dimensional $D=3$ spinorial Lorentz group $\mathrm{SL}(2 ; \mathbb{R}) \approx \overline{\mathrm{SO}(2,1)}$ manifold, with three independent coordinates, two related with the three-momentum on the mass-shell and the third with arbitrary $D=3$ Abelian spin values. After introducing suitable incidence relations we obtain the two-twistor formulation with eight-dimensional phase space restricted by one first-class mass constraint. If we quantize such two-twistorial model we obtain the wave function defined on the $\mathrm{SL}(2 ; \mathbb{R})$ group manifold. After providing the realization of the $D=3$ spin operator we get that the power expansion of the wave function (see (2.45)) provides in momentum space a $D=3$ massive infinite-dimensional multiplet with all values of spin.

In section 3 , the $D=4$ case is considered. First, we provide variables useful in the relativistic kinematics of massive particles with spin (four-momenta, Pauli-Lubański fourvector, orthonormal bases in four-momentum space called also Lorentz harmonics) in terms of two-twistor geometry. Secondly, we consider the extension of the $D=4$ hybrid action to two-twistor space. In the general case, the auxiliary coordinates present in (1.1) can be enlarged by the replacements

$$
\begin{aligned}
& x_{\alpha \dot{\beta}} \rightarrow\left(x_{\alpha \dot{\beta}}, y_{\alpha \dot{\beta}}^{r}\right), \quad y_{\alpha} \rightarrow y_{\alpha}^{i}, \quad r=1,2,3, \quad i, j=1,2, \\
& y_{\alpha \beta} \rightarrow y_{\alpha \beta}^{i j}=y_{\beta \alpha}^{j i}, \quad \bar{y}_{\dot{\alpha} \dot{\beta}} \rightarrow \bar{y}_{\dot{\alpha} \dot{\beta}}^{i j}=\bar{y}_{\dot{\beta} \dot{\alpha}}^{j i} .
\end{aligned}
$$

The standard Shirafuji model with spacetime coordinates $x_{\alpha \dot{\beta}}$ and a pair of spinors $\left(\pi_{\alpha} \rightarrow\right.$ $\pi_{\alpha}^{i}, \bar{\pi}_{\dot{\alpha}} \rightarrow \bar{\pi}_{\dot{\alpha} i}$ ) leads, after using the standard incidence relation (see e.g. [7]), to a twotwistorial $D=4$ free particle model with four first class constraints. If the two spinorial mass constraints

$$
\mathcal{M}=\pi_{\alpha}^{i} \pi_{i}^{\alpha}+2 M=0, \quad \overline{\mathcal{M}}=\bar{\pi}_{\dot{\alpha}}^{i} \bar{\pi}_{i}^{\dot{\alpha}}+2 \bar{M}=0
$$

are further added, where $\pi_{i}^{\alpha}=\epsilon^{\alpha \beta} \epsilon_{i j} \pi_{\beta}^{j}$ and $M$ is a complex mass parameter, ${ }^{3}$ one obtains a model describing $D=4$ spinless massive particle with four first class and two second class

\footnotetext{
${ }^{3}$ It is related with the mass parameter $m$ of the particle through $2|M|^{2}=m^{2}$ (see also (3.6)).
} 
constraints. To modify the constraints that require the spin to be zero, we introduce three additional auxiliary four-vector coordinates $y_{\alpha \dot{\beta}}^{r}(r=1,2,3)$ (see (1.9)). Arranging correctly the generalized incidence relations we obtain the two-twistorial free model with one first class and two second class constraints, which reduce the 16 twistor real coordinates $\left(\pi_{\alpha}^{i}, \bar{\pi}_{\dot{\alpha} i}\right)$ (eq. (3.1)) to 12 physical degrees of freedom. These new versions of the hybrid model can be quantized and solved by using the 'spinorial roots' $\left(\pi_{\alpha}^{i}, \bar{\pi}_{\dot{\alpha} i}\right)$ of the four-momenta as independent variables, which provides the reduced $D=4$ wave function $\psi\left(\pi_{\alpha}^{i}, \bar{\pi}_{\dot{\alpha} i}\right)$. If we take into consideration the mass constraints (1.10) we obtain that the manifold of the spinorial coordinates is described by the group manifold of $\mathrm{SL}(2 ; \mathbb{C})$, the cover of the $D=4$ Lorentz group, with its six real parameters being half of the twelve physical phase space degrees of freedom that are left in the bitwistorial formulation in our model. We show that such a wave function can be identified with the $D=4$ master field describing an infinite-dimensional multiplet of massive HS fields with arbitrary $D=4$ spin spectrum (for an analogy see [53]).

Finally, in section 4 we present some comments going beyond $D=3,4$, on possible $D=6$ and supersymmetric extensions. Further, the Outlook briefly discusses how to introduce nonlinear interactions of the massive HS multiplets and how to adapt, in our massive case, the construction for massless HS fields in [4, 19-21] which uses, in suitably chosen dimensions, the duality between the HS current and HS field multiplets.

The paper includes two appendices. Appendix A details our conventions; appendix B presents an interpretation of our $N=2 D=3$ spinorial model in section 2.2 as described by an $N=1 D=4$ vectorial model with the nonstandard $\mathrm{O}(2,2)$ Lorentz group.

\section{$2 \quad D=3$ bispinorial particle models and HS massive fields from their quantization}

\subsection{Summary of $D=3$ two-twistor kinematics}

$D=3$ twistors are real four-dimensional $\mathrm{Sp}(4 ; \mathbb{R})=\overline{\mathrm{SO}(3,2)}$ spinors. We introduce a pair of $D=3$ real twistors

$$
t^{A i}=\left(\begin{array}{c}
\lambda_{\alpha}^{i} \\
\mu^{\alpha i}
\end{array}\right), \quad \alpha=1,2, \quad i=1,2, \quad A=1, \ldots, 4,
$$

with conformal-invariant scalar product ${ }^{4}$

$$
t_{A}^{i} t_{i}^{A}=t_{A}^{i} \epsilon_{i j} t^{A j}
$$

where the contravariant spinor

$$
t_{A}^{i}=g_{A B} t^{B i}
$$

is constructed using the $\operatorname{Sp}(4 ; \mathbb{R})$-invariant antisymmetric metric (see also footnote 1 )

$$
g_{A B}=\left(\begin{array}{cc}
0 & -\delta^{\alpha}{ }_{\beta} \\
\delta_{\alpha}{ }^{\beta} & 0
\end{array}\right) .
$$

\footnotetext{
${ }^{4}$ The conformal $D=3$ twistors are null twistors.
} 
If we only employ the spinors $\lambda_{\alpha}^{i}$ we can construct the following $D=3$ bilinears describing composite three-vectors in internal $N=2(i, j=1,2)$ space

$$
u_{\alpha \beta}^{a}=\lambda_{\alpha}^{i}\left(\gamma^{a}\right)_{i j} \lambda_{\beta}^{j}, \quad a \equiv(0, r)=(0,1,2),
$$

where the $2 \times 2$ matrices $\left(\gamma^{a}\right)_{i j}$ are internal space $\mathrm{SO}(2,1)$ Dirac matrices (eq. (A.10)) and form a basis for the space of symmetric $2 \times 2$ matrices (see appendix A). Further, according to Penrose twistor theory (see e.g. [7]) we take $u_{\alpha \beta}^{0}=p_{\alpha \beta}$ (three-momentum). We shall further impose the following spinorial mass constraint

$$
\Lambda \equiv \lambda_{\alpha}^{i} \lambda_{i}^{\alpha}+\sqrt{2} m=0, \quad \lambda_{i}^{\alpha}=\epsilon_{i j} \epsilon^{\alpha \beta} \lambda_{\beta}^{j},
$$

which implies that the three-vectors (2.5) describe, after suitable normalization $e_{\alpha \beta}^{a}=$ $\frac{1}{m} u_{\alpha \beta}^{a}$, the $D=3$ vectorial harmonics (see e.g. $[54-56]^{5}$ ) describing the $D=3$ Lorentz orthonormal vector frame

$$
e_{\alpha \beta}^{a} e^{b \alpha \beta}=\eta^{a b}, \quad \eta^{a b}=(1,-1,-1) .
$$

It is easy to check that the set of three-vectors $u_{\alpha \beta}^{a}$ has three independent degrees of freedom equal to the number of spinorial degress of freedom constrained by the relation (2.6). In particular, if $a=b=0$ we obtain from (2.7) the mass-shell condition for the $D=3$ momenta

$$
p_{\alpha \beta} p^{\alpha \beta}=m^{2},
$$

where

$$
p_{\alpha \beta} \equiv u_{\alpha \beta}^{0}=\lambda_{\alpha}^{i} \lambda_{\beta}^{i} .
$$

In order to describe the realizations of Lorentz group and the Abelian scalar $D=3$ spin $S$ we should use all twistor components (see (2.1)). The Lorentz algebra generators $M_{\mu \nu}=L_{\mu \nu}+S_{\mu \nu}, L_{\mu \nu}=x_{\mu} p_{\nu}-x_{\nu} p_{\mu}$ are given in spinorial notation by

$$
M_{\alpha \beta}=-\frac{1}{\sqrt{2}} \lambda_{(\alpha}^{i} \mu_{\beta)}^{i} \equiv-\frac{1}{2 \sqrt{2}}\left(\lambda_{\alpha}^{i} \mu_{\beta}^{i}+\lambda_{\beta}^{i} \mu_{\alpha}^{i}\right)
$$

and the scalar spin $S$ for the massive particle with mass $m$ is described by the $D=3$ counterpart of the Pauli-Lubański operator given by $(\mu, \nu, \varrho=0,1,2)$

$$
\frac{1}{2} \epsilon_{\mu \nu \rho} p^{\mu} M^{\nu \rho}=p^{\mu} M_{\mu}=p^{\alpha \beta} M_{\alpha \beta}=m S, \quad S=\frac{1}{2} \lambda_{\alpha}^{i} \mu_{i}^{\alpha}=\frac{1}{4} t_{A}^{i} t_{i}^{A},
$$

where $M_{\mu}=\frac{1}{2} \epsilon_{\mu \nu \lambda} M^{\nu \lambda}$ and we use the bitwistor representation of momenta (2.9). We see that $D=3$ spin is described by the unique nonvanishing conformal-invariant twistor norm provided by formula $(2.2)$.

We shall further consider the field equations that determine the mass and spin eigenvalues of the $D=3$ Casimirs (2.8) and (2.11). Such field equations were also considered in quantum theory as describing anyons, with arbitrary fractional value of $s$ (see e.g. [57-61]). In the next section we obtain these equations with fixed $m$ and half-integer values of $s$ as a result of the quantization of the new particle action. We will not consider here the anyonic fractional spin values that come from representations of the universal cover $\mathbb{R}$ of the $D=3$ Abelian spin group $\mathrm{U}(1)$.

\footnotetext{
${ }^{5}$ The authors thank Evgeny Ivanov for informing about the reference [56].
} 


\section{2 $D=3$ bispinorial generalization of the Shirafuji model}

We propose the following action for our $D=3$ model $(i, j=1,2 ; r=1,2)$

$$
S^{(3)}=\int d \tau\left[\lambda_{\alpha}^{i} \lambda_{\beta}^{i} \dot{x}^{\alpha \beta}+c \lambda_{\alpha}^{i}\left(\gamma^{r}\right)_{i j} \lambda_{\beta}^{j} \dot{y}_{r}^{\alpha \beta}+f \lambda_{\alpha}^{i} \dot{y}_{i}^{\alpha}+\ell\left(\lambda_{\alpha}^{i} \lambda_{i}^{\alpha}+\sqrt{2} m\right)\right]
$$

where $\lambda_{i}^{\alpha}=\epsilon^{\alpha \beta} \epsilon_{i j} \lambda_{\beta}^{j}$ etc. and $\ell$ is a Lagrange multiplier imposing the constraint $\Lambda$ in eq. (2.6). The parameters $c, f$ may be set equal to one by rescaling the coordinates, but we shall keep them arbitrary in order to consider various variants of the model (actually, the most interesting values are 0 and 1 ). In particular, if we set $c=1$ the first two terms in (2.12) collapse into $\lambda_{\alpha}^{i}\left(\gamma^{a}\right)_{i j} \lambda_{\beta}^{j} \dot{y}_{a}^{\alpha \beta}$ where $y_{a}^{\alpha \beta}=\left(\dot{x}^{\alpha \beta}, y_{r}^{\alpha \beta}\right)$ with $a=(0, r)=(0,1,2)$. If $c=f=0$, after using the standard incidence relation

$$
\mu^{\alpha i}=2 x^{\alpha \beta} \lambda_{\beta}^{i},
$$

and inserting (2.13) into (2.11), we get $S=0$, i.e. we obtain the model describing a spinless particle. In the general case the incidence relation (2.13) has to be generalized as follows ${ }^{6}$

$$
\mu^{\alpha i}=2 x^{\alpha \beta} \lambda_{\beta}^{i}+2 c\left(\gamma^{r}\right)_{i j} y_{r}^{\alpha \beta} \lambda_{\beta}^{j}+f y^{\alpha i} .
$$

After using relations (2.5) in (2.11) we obtain

$$
S=-c \lambda_{\alpha}{ }^{i}\left(\gamma^{r}\right)_{j}{ }^{i} y_{r}^{\alpha \beta} \lambda_{\beta}^{j}-\frac{1}{2} f \lambda_{\alpha i} y^{\alpha i},
$$

thus, $S \neq 0$ whenever $c$ or $f$ are non-zero.

Setting $c=f=1$, the constraints defining the momenta follow from (2.12) with the result

$$
\begin{aligned}
T_{\alpha \beta}^{a}=T_{\beta \alpha}^{a} & \equiv p_{\alpha \beta}^{a}-u_{\alpha \beta}^{a} \approx 0, \\
G_{\alpha}^{i} & \equiv p_{(y)_{\alpha}}{ }^{i}-\lambda_{\alpha}^{i} \approx 0, \\
F_{i}^{\alpha} & \equiv p_{(\lambda) i}^{\alpha} \approx 0 .
\end{aligned}
$$

Eqs. (2.17) and (2.18) determine pairs of second class constraints. After introducing for them Dirac brackets we obtain that the variables $\left(y_{a}^{\alpha \beta}, y_{i}^{\alpha}\right), a=0,1,2$, are canonically conjugate to $\left(p_{\alpha \beta}^{a}, \lambda_{i}^{\alpha}\right)$ so that the non-vanishing $\mathrm{PBs}$ are given by

$$
\begin{aligned}
\left\{y_{a}^{\alpha \beta}, p_{\gamma \delta}^{b}\right\} & =\delta_{a}^{b} \delta_{\gamma}^{(\alpha} \delta_{\delta}^{\beta)}, \\
\left\{y_{i}^{\alpha}, \lambda_{\beta}^{j}\right\} & =\delta_{i}^{j} \delta_{\beta}^{\alpha},
\end{aligned}
$$

where we recall that $A^{(\alpha} B^{\beta)} \equiv \frac{1}{2}\left(A^{\alpha} B^{\beta}+A^{\beta} B^{\alpha}\right)$.

The model (2.12) has ten first class constraints expressed by the formula (2.16) and the mass-shell constraint (2.6). After quantization the above PB relations can be realized in terms of $\hat{y}_{a}^{\alpha \beta}=y_{a}^{\alpha \beta}, \hat{\lambda}_{\alpha}^{i}=\lambda_{\alpha}^{i}$ and the following differential operators

$$
\hat{p}_{\alpha \beta}^{a}=-i \frac{\partial}{\partial y_{a}^{\alpha \beta}}, \quad \hat{y}_{i}^{\alpha}=i \frac{\partial}{\partial \lambda_{\alpha}^{i}},
$$

\footnotetext{
${ }^{6}$ Relation (2.14) is adjusted in order to obtain from (2.12) the free two-twistor action (see section 2.3).
} 
where, by definition, $\frac{\partial}{\partial y_{a}^{\alpha \beta}} y_{b}^{\gamma \delta}=\delta_{b}^{a} \delta_{\alpha}^{(\gamma} \delta_{\beta}^{\delta)}$. As a result, the quantized constraints (2.16) after using equations (2.5) deterimine the following three unfolded equations for the wave function $\Phi \equiv \Phi\left(y_{a}^{\alpha \beta}, \lambda_{i}^{\alpha}\right)$,

$$
\left(i \frac{\partial}{\partial y_{a}^{\alpha \beta}}+\lambda_{\alpha}^{i}\left(\gamma^{a}\right)_{i j} \lambda_{\beta}^{j}\right) \Phi\left(y_{a}^{\alpha \beta}, \lambda_{i}^{\alpha}\right)=0, \quad a=(0,1,2),
$$

with the following solution expressing explicitly the dependence on $y_{a}^{\alpha \beta}$,

$$
\Phi\left(y_{a}^{\alpha \beta}, \lambda_{\alpha}^{i}\right)=\exp \left\{i \lambda_{\alpha}^{i}\left(\gamma^{a}\right)_{i j} \lambda_{\beta}^{j} y_{a}^{\alpha \beta}\right\} \phi\left(\lambda_{\beta}^{i}\right) .
$$

Using, instead of (2.21), the dual differential realization in spinorial sector

$$
\widehat{\lambda}_{\alpha}^{i}=-i \frac{\partial}{\partial y_{i}^{\alpha}},
$$

one obtains from (2.6) a single field equation for the reduced wave function

$$
\left(\frac{\partial}{\partial y_{i}^{\alpha}} \frac{\partial}{\partial y_{\alpha}^{i}}-\sqrt{2} m\right) \tilde{\phi}\left(y_{i}^{\alpha}\right)=0
$$

where

$$
\tilde{\phi}\left(y_{i}^{\beta}\right)=\int d^{4} \lambda e^{i \lambda_{\alpha}^{i} y_{i}^{\alpha}} \phi\left(\lambda_{\alpha}^{i}\right)
$$

In the 'spinorial momentum' picture described by the spinors $\lambda_{\alpha}^{i}$ the reduced wave function $\phi\left(\lambda_{\alpha}^{i}\right)$ depends on the spinorial momenta restricted by the algebraic equation (2.6). We see that the wave function describing the quantum mechanical solution of the model (2.12) depends on three degrees of freedom, two describing the on-shell three-momenta and a third one being the (arbitrary) value of the $D=3$ spin. In order to express the spin operator (2.11) as a differential operator in spinorial momentum space one has to consider the quantum version of the twistorial description of model (2.12).

Let us now compare the models (2.12) with $f=0$ and $f \neq 0$ (for simplicity we set $c=1$ ). From expression (2.15) it follows that in both models $S$ is a composite dynamical variable that describes arbitrary $D=3$ spin; however, the limit $f \rightarrow 0$ changes the structure of the constraints. Indeed, if $f=0$, those in (2.17) are not present; only the constraints (2.16), (2.18) and the mass-shell constraint (2.6) appear. The alternative constraint structure is well illustrated if the nine relations (2.16) are replaced by the equivalent set of nine Abelian constraints

$$
T_{b}^{a} \equiv T_{\alpha \beta}^{a} u_{b}^{\alpha \beta}=p_{\alpha \beta}^{a} u_{b}^{\alpha \beta}-m^{2} \delta_{b}^{a} \approx 0 .
$$

Similarly, the four constraints (2.18) can be replaced by four equivalent ones as follows

$$
\begin{aligned}
F & =\frac{1}{2} \lambda_{\alpha}^{i} p_{(\lambda) i}^{\alpha} \approx 0, \\
F_{a} & =\frac{1}{2} \lambda_{\alpha}^{i}\left(\gamma_{a}\right)_{i}^{j} p_{(\lambda)}{ }_{j}^{\alpha} \approx 0,
\end{aligned}
$$

where the $D=3$ gamma matrices $\left(\gamma_{a}\right)_{i}{ }^{j}$ satisfy the $s o(1,2)$ commutation relations

$$
\left[\gamma_{a}, \gamma_{b}\right]=-2 \epsilon_{a b}{ }^{c} \gamma_{c},
$$


with metric $\operatorname{diag}(1,-1,-1)$ raising the $\mathrm{O}(2,1)$ indices. Using the canonical $\mathrm{PB}$ $\left\{\lambda_{\alpha}^{i}, p_{(\lambda)}^{\beta}\right\}=\delta_{\alpha}^{\beta} \delta_{j}^{i}$, it is seen that the thirteen new constraints $\left(T_{a}^{b}, F_{a}, F\right)$ have the following non-vanishing PBs:

$$
\begin{aligned}
\left\{F_{a}, F_{b}\right\} & =\epsilon_{a b}^{c} F_{c}, \\
\left\{F_{a}, T_{b}^{c}\right\} & =\epsilon_{a b}{ }^{d} T_{d}^{c}+m^{2} \epsilon_{a b}^{c}, \\
\left\{F, T_{a}^{b}\right\} & =-T_{a}^{b}-m^{2} \delta_{a}^{b}, \\
\{F, \Lambda\} & =-\Lambda+\sqrt{2} m .
\end{aligned}
$$

We see from the second and fourth equations of (2.30) that four out of the ten first class constraints $T_{a}^{b}$ and $\Lambda$ (eq. (2.6)) present when $f \neq 0$ become second class due to the appearance of the four constraints $(2.28)$ in the limit $f=0$. These four constraints $\left(F_{a}, F\right)$ are second class and describe the gauge fixing of four gauge transformations present if $f \neq 0$. We can conclude that putting $f=0$ in (2.12) leads to the partial gauge fixing of four out of the ten gauge degrees of freedom generated when $f \neq 0$ by the ten first class constraints $T_{\alpha \beta}^{a}$ (or $T_{b}^{a}$ ) and $\Lambda$. If $f \neq 0$ the ten first class constraints remove $2 \times 10=20$ real degrees of freedom; for $f=0$ the six first class constraints plus the eight second class remove the same number of d.o.f., $2 \times 6+8=20$. Thus, both models have the same physical (i.e. without gauge degrees of freedom) content. This proves the equivalence of the classical models considered for $f \neq 0$ and $f=0$.

Finally, we point out that for $c=1$ our model (2.12) describes a vectorial $\mathrm{SO}(2,2)$ particle model, as discussed in appendix B.

\section{3 $D=3$ bitwistorial description}

In order to introduce the twistor coordinates (2.1), we insert in (2.12) the generalized incidence relation (2.14). Modulo boundary terms, we obtain for $c \neq 0$ and/or $f \neq 0$ the following twistorial free action with $\operatorname{Sp}(4, \mathbb{R}) D=3$ twistorial metric (1.5):

$$
\tilde{S}^{(3)}=\int d \tau\left[\lambda_{\alpha}^{i} \dot{\mu}^{\alpha i}+\ell\left(\lambda_{\alpha}^{i} \lambda_{i}^{\alpha}+\sqrt{2} m\right)\right] .
$$

The action (2.31) describes an infinite tower of $D=3$ free massive particles with any spin (see e.g. [43]). Let us prove it.

The action (2.31) describes a system with canonical variables $\mu^{\alpha i}$ and $\lambda_{\alpha}^{i},\left\{\mu^{\alpha i}, \lambda_{\beta}^{j}\right\}=$ $\delta^{i j} \delta_{\beta}^{\alpha}$, and the constraint (2.6) which generates the gauge transformations in bitwistor space. Let us fix this gauge freedom by the constraint

$$
G=\lambda_{\alpha}^{i} \mu^{\alpha i} \approx 0, \quad\{\Lambda, G\}=2 \sqrt{2} m-2 \Lambda .
$$

Introducing Dirac brackets incorporating the constraints $\Lambda \approx 0$ and $G \approx 0$ we obtain that they become strong and we get the following Dirac brackets for the twistor variables

$$
\begin{aligned}
\left\{\lambda_{\alpha}^{i}, \lambda_{\beta}^{j}\right\}_{*} & =0 \\
\left\{\mu^{\alpha i}, \lambda_{\beta}^{j}\right\}_{*} & =\delta^{i j} \delta_{\beta}^{\alpha}+\frac{1}{\sqrt{2} m} \lambda_{i}^{\alpha} \lambda_{\beta}^{j}, \\
\left\{\mu^{\alpha i}, \mu^{\beta j}\right\}_{*} & =-\frac{1}{\sqrt{2} m}\left(\lambda_{i}^{\alpha} \mu^{\beta j}-\lambda_{j}^{\beta} \mu^{\alpha i}\right) .
\end{aligned}
$$


A quantum realization of the algebra (2.33) with $\hat{\lambda} \hat{\mu}$ ordering is the following

$$
\hat{\lambda}_{\alpha}^{i}=\lambda_{\alpha}^{i}, \quad \hat{\mu}^{\alpha i}=i \frac{\partial}{\partial \lambda_{\alpha}^{i}}+\frac{i}{\sqrt{2} m} \lambda_{i}^{\alpha} \lambda_{\beta}^{j} \frac{\partial}{\partial \lambda_{\beta}^{j}} .
$$

We point out that the second class constraints (2.6) and (2.32) are fulfilled in the strong sense, i.e. $\hat{G}=\hat{\lambda}_{\alpha}^{i} \hat{\mu}^{\alpha i} \equiv 0$. If we use the formulae (2.34), the spin operator (2.11) is realized as follows

$$
\hat{S}=\frac{1}{2} \hat{\lambda}_{\alpha}^{i} \hat{\mu}_{i}^{\alpha}=\frac{i}{2} \epsilon_{i j} \lambda_{\alpha}^{i} \frac{\partial}{\partial \lambda_{\alpha}^{j}} .
$$

Our aim will be to decompose the Fourier transform (2.26) of the reduced wave function $\tilde{\phi}\left(y_{i}^{\alpha}\right)$ satisfying eq. (2.25) into a superposition of momentum-dependent eigenfunctions of the operator (2.35) (see eqs. (2.53), (2.54) below).

Due to the mass constraint (2.6), the real $2 \times 2$ matrices $h$ with elements

$$
h_{\alpha}{ }^{i}=2^{1 / 4} m^{-1 / 2} \lambda_{\alpha}{ }^{i}
$$

have determinant equal to one, characterize the $\mathrm{SL}(2 ; \mathbb{R})$ group manifold and describe real spinorial $D=3$ harmonics [56] (note the algebra isomorphisms $\operatorname{sl}(2 ; \mathbb{R}) \sim \operatorname{su}(1,1) \sim$ $s p(2 ; \mathbb{R}))$. The corresponding $\mathrm{SU}(1,1)$ matrix is obtained by the complex similarity transformation

$$
g=U h U^{-1}, \quad U=e^{-i \pi \sigma_{1} / 4}
$$

with matrix elements

$$
g=\left(\begin{array}{cc}
a & \bar{b} \\
b & \bar{a}
\end{array}\right), \quad|a|^{2}-|b|^{2}=1, \quad g \in \mathrm{SU}(1,1),
$$

where

$$
a=\frac{1}{2}\left[h_{1}{ }^{1}+h_{2}^{2}+i\left(h_{1}{ }^{2}-h_{2}{ }^{1}\right)\right], \quad b=\frac{1}{2}\left[h_{1}{ }^{2}+h_{2}{ }^{1}-i\left(h_{1}{ }^{1}-h_{2}{ }^{2}\right)\right] .
$$

In terms of the variables (2.39) the spin operator (2.35) takes the form

$$
\hat{S}=-\frac{1}{2}\left(a \frac{\partial}{\partial a}+b \frac{\partial}{\partial b}-\bar{a} \frac{\partial}{\partial \bar{a}}-\bar{b} \frac{\partial}{\partial \bar{b}}\right)
$$

The matrix $g_{\alpha}{ }^{i}$ in $(2.38)$ describes $\mathrm{SU}(1,1)$ spinorial harmonics, where first column $g_{\alpha}{ }^{1}=$ $\left(\begin{array}{l}a \\ b\end{array}\right)$ (second $g_{\alpha}{ }^{2}=\left(\begin{array}{l}\bar{b} \\ \bar{a}\end{array}\right)$ ) describes a SU $(1,1)$ spinor with spin eigenvalue $s=-\frac{1}{2}\left(s=\frac{1}{2}\right)$.

One can introduce the natural parametrization of the $\mathrm{SU}(1,1)$ matrices (2.38) [62]

$$
a=\cosh (r / 2) e^{i(\psi+\varphi) / 2}, \quad b=\sinh (r / 2) e^{i(\psi-\varphi) / 2},
$$

where

$$
0 \leq \varphi \leq 2 \pi, \quad 0<r<\infty, \quad-2 \pi \leq \psi<2 \pi .
$$

In terms of the angle $\psi$, the operator (2.40) takes the simple form

$$
\hat{S}=i \frac{\partial}{\partial \psi}
$$

i.e., it describes the $D=3 \mathrm{U}(1)$ spin. 
After the transformation (2.36), the twistorial wave function $\Psi(g)$ is defined on $\mathrm{SU}(1,1)$. The $\mathrm{SU}(1,1)$ regular representation is given by its action of on the (wave) functions $\Psi(g)$ defined on the $\mathrm{SU}(1,1)$ manifold. To obtain the Hilbert space of the quantized model (2.31) we may use the theory of special functions on matrix group manifolds (see e.g. [62]) and require that the wave function $\Psi(g)=\Psi(\varphi, r, \psi)$ is square-integrable, $\int|\Psi(g)|^{2} d g<+\infty, d g=\sinh r d r d \varphi d \psi$. Due to eq. (2.41), the wave function satisfies the periodicity conditions

$$
\Psi(\varphi, r, \psi)=\Psi(\varphi+4 \pi, r, \psi)=\Psi(\varphi, r, \psi+4 \pi)=\Psi(\varphi+2 \pi, r, \psi+2 \pi),
$$

which eliminate the anyonic quantum states with arbitrary fractional spin.

One can use the double Fourier expansion

$$
\Psi(\varphi, r, \psi)=\sum_{k, n=-\infty}^{\infty} f_{k n}(r) e^{-i(k \varphi+n \psi)}=\sum_{n=-\infty}^{\infty} e^{-i n \psi} F_{n}(r, \varphi),
$$

where $F_{n}(r, \varphi) \equiv \sum_{k=-\infty}^{\infty} f_{k n}(r) e^{i k \varphi}$ ( $n$ is fixed). The summation is over all pairs $(k, n)$ such that the numbers $k$ and $n$ are both integer or half-integer. The eigenvalues of the operator $\hat{S}$ defined by (2.43) coincide with parameter $n$ in the expansion (2.45). As a result, the spin in our model takes quantized integer and half-integer values. The functions $F_{n}(r, \varphi)$ describe states with definite $D=3$ spin equal to $n$. The $r$-dependent fields in (2.45) are expressed by

$$
f_{k n}(r)=\frac{1}{8 \pi^{2}} \int_{-2 \pi}^{2 \pi} \int_{0}^{2 \pi} d \varphi d \psi e^{i(k \varphi+n \psi)} \Psi(\varphi, r, \psi)
$$

and the Plancherel formula gives

$$
\frac{1}{8 \pi^{2}} \int_{-2 \pi}^{2 \pi} \int_{0}^{2 \pi} \int_{0}^{\infty} d \varphi d \psi d r|\Psi(\varphi, r, \psi)|^{2} \sinh r=\sum_{k, n=-\infty}^{\infty} \int_{0}^{\infty} d r\left|f_{k n}(r)\right|^{2} \sinh r .
$$

Square integrable functions $f_{k n}(r)$ have an (integral) expansion on the matrix elements of the SU(1,1) infinite-dimensional unitary representations (see $[62,63]$ for details). Using (2.9) and (2.36), (2.41) we obtain that

$$
\begin{aligned}
& p_{0}=m(a \bar{a}+b \bar{b})=m \cosh r, \\
& p_{1}=i m(a \bar{b}-b \bar{a})=-m \sinh r \sin \varphi, \\
& p_{2}=m(a \bar{b}+b \bar{a})=m \sinh r \cos \varphi ;
\end{aligned}
$$

where $p_{0}^{2}-p_{1}^{2}-p_{2}^{2}=m^{2}$. We see that the on-shell momentum components (2.48) do not depend on the angle $\psi$ and thus define the coset manifold $\mathrm{SU}(1,1) / \mathrm{U}(1)$, the hyperboloid which is the base manifold of the (trivial) $\mathrm{U}(1)$-fibration of $\mathrm{SU}(1,1)$. The wave function (2.44) with the Fourier expansion (2.45) in the U(1) $\psi$-variable describes an infinitedimensional tower of $D=3$ higher spin fields. 
The coefficient fields in the expansion in (2.45) are defined on the coset $\mathrm{SU}(1,1) / \mathrm{U}(1)$ as functions of the on-shell three-momenta $p_{\mu}$,

$$
F_{n}(r, \varphi)=\tilde{F}_{n}\left(p_{\mu} ; m\right)
$$

and

$$
f_{k n}(r)=\tilde{f}_{k n}\left(p_{0} ; m\right)
$$

Let us analyze the expansion (2.45) in a Lorentz covariant form.

We recall that the transformation (2.37) describes the isomorphism between $\operatorname{SL}(2 ; \mathbb{R})$ and $\mathrm{SU}(1,1)$ matrix group (see, for example, [64]). Using eq. (2.37) one can transform $D=$ 3 spinors and $\gamma$-matrices from Majorana (real) representation to a complex representation. We get in such a way the $D=3$ framework which uses the $\mathrm{SU}(1,1)$ spinor coordinates ${ }^{7}$

$$
\xi_{\alpha}=\sqrt{m}\left(\begin{array}{l}
a \\
b
\end{array}\right), \quad \bar{\xi}^{\alpha}=\left(\xi_{\alpha}\right)^{\dagger}=\sqrt{m}(\bar{a}, \bar{b}), \quad \bar{\xi}^{\alpha}\left(\sigma_{3}\right)_{\alpha}{ }^{\beta} \xi_{\beta}=m .
$$

In the variables (2.51) the $D=3$ spin operator (2.40) takes the form

$$
\hat{S}=\frac{1}{2}\left(\bar{\xi}^{\alpha} \frac{\partial}{\partial \bar{\xi}^{\alpha}}-\xi_{\alpha} \frac{\partial}{\partial \xi_{\alpha}}\right) .
$$

We find easily that in terms of the $\mathrm{SU}(1,1)$ spinors $(2.51)$ the three-momentum $(2.48)$ is given by

$$
p_{\mu}=\widetilde{\xi}^{\alpha}\left(\gamma_{\mu}\right)_{\alpha \beta} \xi^{\beta},
$$

where $\widetilde{\xi}^{\alpha}=\bar{\xi}^{\beta}\left(\gamma_{0}\right)_{\beta}{ }^{\alpha}$ is the Dirac conjugated spinor, $\xi^{\beta}=\epsilon^{\beta \alpha} \xi_{\alpha},\left(\gamma_{\mu}\right)_{\alpha}{ }^{\beta}$ are Dirac $\gamma$ matrices in the complex SU(1,1) representation (A.7), $\left(\gamma_{\mu}\right)_{\alpha \beta}=\epsilon_{\beta \gamma}\left(\gamma_{\mu}\right)_{\alpha}{ }^{\gamma}$ and $p_{\mu}=$ $\widetilde{\xi}^{\alpha}\left(\gamma_{\mu}\right)_{\alpha \beta} \xi^{\beta}=-\widetilde{\xi}^{\alpha}\left(\gamma_{\mu}\right)_{\alpha}{ }^{\beta} \xi_{\beta} \equiv-\tilde{\xi} \gamma_{\mu} \xi$. Eq. (2.53) is the $D=3$ counterpart of the standard Penrose formula for the four-momenta in the $D=4$ case, in which the $D=4 \mathrm{SL}(2 ; \mathbb{C})$ Weyl spinors have been replaced by $D=3 \mathrm{SU}(1,1)$ spinors.

Using relations (2.41) and (2.51) we can write down the expansion (2.45) in the covariant form

$$
\Psi(\xi, \bar{\xi})=\sum_{N, K=0}^{\infty} \xi_{\alpha_{1}} \ldots \xi_{\alpha_{K}} \bar{\xi}^{\beta_{1}} \ldots \bar{\xi}^{\beta_{N}} \psi_{\beta_{1} \ldots \beta_{N}}^{\alpha_{1} \ldots \alpha_{K}}\left(p_{\mu}\right),
$$

where $\psi_{\beta_{1} \ldots \beta_{N}}^{\alpha_{1} \ldots \alpha_{K}}\left(p_{\mu}\right)$ is the covariant counterpart of the functions $\tilde{F}_{n}\left(p_{\mu} ; m\right)$ where $\frac{N-K}{2}=n$ (see eqs. (2.54), (2.51), (2.41) and (2.45)).

We note that the $\mathrm{SU}(1,1)$ spinorial formalism is more convenient for the description of spin states than the $\mathrm{SL}(2 ; \mathbb{R})$ framework because it diagonalizes the spin eigenvalues. Formally the wave function (2.54) (or the reduced wave function $\phi\left(\lambda_{\alpha}^{i}\right)$ in (2.26)), after using (2.6), can be written as follows

$$
\phi(\lambda)=\sum_{N=0}^{\infty} \lambda_{\alpha_{1}}^{i_{1}} \ldots \lambda_{\alpha_{N}}^{i_{N}} \tilde{\psi}_{i_{1} \ldots i_{N}}^{\alpha_{1} \ldots \alpha_{N}}\left(p_{\mu}\right) .
$$

However, the monomials $\lambda_{\alpha_{1}}^{i_{1}} \ldots \lambda_{\alpha_{N}}^{i_{N}}$ are not eigenvectors of the spin operator (2.35).

\footnotetext{
${ }^{7}$ We use the index $\alpha=1,2$ for the real $\mathrm{SL}(2 ; \mathbb{R})$ as well as for the complex $\mathrm{SU}(1,1)$ spinors since it is a Dirac spinor index in different realizations of the $D=3 \gamma$-matrices. Note that the reality of a $\operatorname{SL}(2 ; \mathbb{R})$ spinor $\chi=\bar{\chi}$ implies the validity of $D=3 \mathrm{SU}(1,1)$ Majorana condition $\psi^{\dagger} \gamma_{0}=\psi^{T} C$ for the $\mathrm{SU}(1,1)$ spinor $\psi=U \chi$, where in accordance with (A.7) $\gamma_{0}=i \sigma_{3}, C=i \sigma_{2}$.
} 
We point out that the expansions (2.54) include both states with positive $(n>0)$ and negative $(n<0)$ spin values and that it is infinitely degenerate because a spin $n$ is generated by all monomials $\xi_{\alpha_{1}} \ldots \xi_{\alpha_{K}} \bar{\xi}^{\beta_{1}} \ldots \bar{\xi}^{\beta_{N}}$ such that $n=\frac{N-K}{2}$. One can remove the degeneracy in $N, K$ for a given $n$ by projecting on the spaces with definite sign of spin if we consider anti-holomorphic wave functions satisfying the condition

$$
\frac{\partial}{\partial \xi_{\alpha}} \Psi(\xi, \bar{\xi})=0
$$

A solution of (2.56) is provided by the power series

$$
\Psi^{(+)}(\bar{\xi})=\sum_{N=0}^{\infty} \bar{\xi}^{\alpha_{1}} \ldots \bar{\xi}^{\alpha_{N}} \psi^{(+)}{ }_{\alpha_{1} \ldots \alpha_{N}}\left(p_{\mu}\right),
$$

which depends only on $\bar{\xi}$ and contains only positive spins.

Alternatively, we may impose the condition

$$
\frac{\partial}{\partial \bar{\xi}^{\alpha}} \Psi(\xi, \bar{\xi})=0
$$

which can also be interpreted as another $\mathrm{SU}(1,1)$ harmonic expansion condition.

The spacetime dependent fields are obtained in the standard way by means of a generalized Fourier transform with exponent $e^{i p_{\mu} x^{\mu}}=e^{-i\left(\tilde{\xi} \gamma_{\mu} \xi\right) x^{\mu}}$ and measure $\mu^{3}(\xi)=$ $d^{4} \xi \delta\left(\bar{\xi} \sigma_{3} \xi-m\right)$ (see eq. (2.51)). We get in such a way the Fourier-twistor transform for $D=3$ massive fields. The corresponding spacetime fields are then given by

$$
\phi_{\alpha_{1} \ldots \alpha_{N}}^{(+)}(x)=\int \mu^{3}(\xi) e^{-i\left(\tilde{\xi} \gamma_{\mu} \xi\right) x^{\mu}} \xi_{\alpha_{1}} \ldots \xi_{\alpha_{N}} \Psi^{(+)}(\xi) .
$$

The fields (2.59) are symmetric with respect to their spinorial indices and satisfy the $D=3$ Bargmann-Wigner equations

$$
\partial_{\mu}\left(\gamma^{\mu}\right)_{\beta}^{\alpha_{1}} \phi_{\alpha_{1} \alpha_{2} \ldots \alpha_{N}}^{(+)}-m \phi_{\beta \alpha_{2} \ldots \alpha_{N}}^{(+)}=0
$$

where the $\gamma$-matrices are taken in the complex $\mathrm{SU}(1,1)$ representation (A.7).

The negative $(n<0)$ spin (helicity) states are described by the holomorphic twistor wave function

$$
\Psi^{(-)}(\xi)=\sum_{N=0}^{\infty} \xi_{\alpha_{1}} \ldots \xi_{\alpha_{N}} \psi^{(-) \alpha_{1} \ldots \alpha_{N}}\left(p_{\mu}\right),
$$

which is a solution of equation (2.58). The twistor transform can be obtained by the complex conjugation of $(2.59)$

$$
\phi^{(-) \alpha_{1} \ldots \alpha_{N}}(x)=\int \mu^{3}(\xi) e^{i\left(\tilde{\xi} \gamma_{\mu} \xi\right) x^{\mu}} \bar{\xi}^{\alpha_{1}} \ldots \bar{\xi}^{\alpha_{N}} \Psi^{(-)}(\xi)
$$

and defines spacetime fields with symmetric spinorial indices that satisfy the BargmannWigner equations (2.60) with $m \rightarrow-m$. 


\section{$3 \quad D=4$ bispinorial models and HS massive fields}

\subsection{Summary of $D=4$ two-twistor kinematics}

The standard $D=4$ Penrose twistors are complex four-dimensional $\mathrm{SU}(2,2)=\overline{\mathrm{SO}(4,2)}$ spinors $Z^{A i}, \bar{Z}_{A i}$ that can be expressed by two pairs of two-component Weyl spinors $\left(\pi_{\alpha}^{i}, \bar{\omega}^{\dot{\alpha} i}\right)$

$$
Z^{A i}=\left(\begin{array}{c}
\pi_{\alpha}^{i} \\
\bar{\omega}^{\dot{\alpha} i}
\end{array}\right), \quad\left(Z^{A i}\right)^{*} \equiv\left(\begin{array}{c}
\bar{\pi}_{\dot{\alpha} i} \\
\omega_{i}^{\alpha}
\end{array}\right), \quad \bar{Z}_{A i}=\left(\omega_{i}^{\alpha},-\bar{\pi}_{\dot{\alpha} i}\right)
$$

where $\bar{\pi}_{\dot{\alpha} i}=\left(\pi_{\alpha}^{i}\right)^{*}, \omega_{i}^{\alpha}=\left(\bar{\omega}^{\dot{\alpha} i}\right)^{*}$. One can introduce four conformal-invariant scalar products $(a=0,1,2,3)$

$$
S_{i}{ }^{j}=\bar{Z}_{A i} Z^{A j} \quad \text { or } \quad S^{a}=Z^{A i}\left(\sigma^{a}\right)_{i}{ }^{j} \bar{Z}_{A j}
$$

where the hermitian $2 \times 2$ matrices above $\sigma^{a}$ are defined in appendix $\mathrm{A}$ and act in the internal bidimensional space.

Using the complex Weyl spinors $\pi_{\alpha}^{i}, \bar{\pi}_{\dot{\alpha} i}$ we can define the following set of real composite four-vectors

$$
u_{\alpha \dot{\beta}}^{a}=\pi_{\alpha}^{i}\left(\sigma^{a}\right)_{i}{ }^{j} \bar{\pi}_{\dot{\beta} j}, \quad a=0,1,2,3,
$$

which for $a=0$ give the Penrose formula for the composite four-momentum [7]

$$
u_{\alpha \dot{\beta}}^{0} \equiv p_{\alpha \dot{\beta}}=\pi_{\alpha}^{i} \bar{\pi}_{\dot{\beta} i} \equiv \frac{1}{\sqrt{2}} \sigma_{\alpha \dot{\beta}}^{\mu} p_{\mu} .
$$

We shall impose (see (1.10)) two complex spinorial mass constraints by means of the complex mass parameter $M=M_{1}+i M_{2}$. From (3.4) and (1.10) it follows easily that

$$
p_{\alpha \dot{\beta}} p^{\alpha \dot{\beta}}=p_{\mu} p^{\mu}=2|M|^{2},
$$

i.e.

$$
|M|^{2}=\frac{1}{2} m^{2},
$$

where $m$ is the mass of the particle. Using further the real four-vector notation

$$
u_{\mu}^{a}=\frac{1}{\sqrt{2}}\left(\sigma_{\mu}\right)^{\alpha \dot{\beta}} u_{\alpha \dot{\beta}}^{a}, \quad e_{\mu}^{a}=\frac{1}{m} u_{\mu}^{a},
$$

it follows that (cf. (2.7))

$$
u_{\mu a} u_{b}^{\mu}=m^{2} \eta_{a b}, \quad e_{\mu a} e^{\mu}{ }_{b}=\eta_{a b}, \quad \eta_{a b}=(1,-1,-1,-1) .
$$

The four-vectors $e_{\mu}^{a}$ in eqs. (3.7), (3.8) describe an orthonormal vectorial Lorentz frame defining $D=4$ vectorial Lorentz harmonics; the spinors $\sqrt{\frac{2}{m}} \pi_{\alpha}^{i}, \sqrt{\frac{2}{m}} \bar{\pi}_{\dot{\alpha} i}$ constitute a pair of complex-conjugated spinorial $D=4$ Lorentz harmonics [65-67].

The two-twistorial realization of the $D=4$ Poincaré algebra $P_{\mu} \simeq P_{\alpha \dot{\beta}}, M_{\mu \nu} \simeq$ $\left(M_{\alpha \beta}, M_{\dot{\alpha} \dot{\beta}}\right)$ can be expressed in terms of the twistor components (eq. (3.1)) as follows ${ }^{8}[7]$.

$$
P_{\alpha \dot{\beta}}=\pi_{\alpha}^{i} \bar{\pi}_{\dot{\beta} i}, \quad M_{\alpha \beta}=\pi_{(\alpha}^{i} \omega_{\beta) i}, \quad M_{\dot{\alpha} \dot{\beta}}=\bar{\omega}_{(\dot{\alpha}}^{i} \bar{\pi}_{\dot{\beta}) i} .
$$

\footnotetext{
${ }^{8}$ In (3.9) we assume the canonical quantization rules for the twistor variables; see also section 3.3.
} 
The Pauli-Lubański four-vector $W_{\mu}$ describing the $D=4$ relativistic spin,

$$
W_{\mu}=\frac{1}{2} \epsilon_{\mu \nu \rho \sigma} P^{\nu} M^{\rho \sigma},
$$

can be written after using expressions (3.9) and (3.2) as an expression in twistorial coordinates as follows

$$
W^{\alpha \dot{\beta}}=S_{r} u_{r}^{\alpha \dot{\beta}}, \quad r=1,2,3,
$$

where

$$
S_{r}=-\frac{i}{2}\left(\pi_{\alpha}^{i} \omega_{j}^{\alpha}-\bar{\pi}_{\dot{\alpha} j} \bar{\omega}^{\dot{\alpha} i}\right)\left(\sigma_{r}\right)_{i}{ }^{j}, \quad r=1,2,3 .
$$

Further, using the relations (1.10), (3.5) and (3.6) it follows that

$$
W^{\mu} W_{\mu}=-m^{2} \vec{S}^{2}, \quad \vec{S}^{2} \equiv S_{r} S_{r} .
$$

After quantization, as it is shown in section 3, we obtain the well known relativistic spin square spectrum with $\vec{S}^{2}$ replaced by $s(s+1)\left(s=0, \frac{1}{2}, 1, \ldots\right)$. We observe that the covariant generators $S_{r}$, which (see (3.11) and (3.8)) can be expressed as

$$
S_{r}=-\frac{1}{m^{2}} u_{r}^{\alpha \dot{\beta}} W_{\alpha \dot{\beta}}
$$

and describe the $s u(2)$ spin algebra in a Lorentz frame-independent way.

\section{2 $D=4$ bispinorial generalization of Shirafuji model}

Following the choice made in the $D=3$ case (see (2.12)), we shall generalize the standard $D=4$ bispinor Shirafuji action by adding three additional terms depending on the supplementary four-vectors $y_{r}^{\mu}(r=1,2,3)$ and on the spinorial kinetic terms, plus the pair of spinorial mass shell constraints $\mathcal{M}, \overline{\mathcal{M}}$ in eq. (1.10):

$$
\begin{gathered}
S^{(4)}=\int d \tau\left[\pi_{\alpha}^{i} \bar{\pi}_{\dot{\beta} i} \dot{x}^{\alpha \dot{\beta}}+c \pi_{\alpha}^{i}\left(\sigma^{r}\right)_{i}{ }^{j} \bar{\pi}_{\dot{\beta} j} \dot{y}_{r}^{\alpha \dot{\beta}}+f \pi_{\alpha}^{i} \dot{y}_{i}^{\alpha}+\bar{f} \bar{\pi}_{\dot{\alpha} i} \dot{\bar{y}}^{\alpha i}\right. \\
\left.+\rho\left(\pi_{\alpha}^{i} \pi_{i}^{\alpha}+2 M\right)+\bar{\rho}\left(\bar{\pi}_{\dot{\alpha}}^{i} \bar{\pi}_{i}^{\dot{\alpha}}+2 \bar{M}\right)\right] .
\end{gathered}
$$

In (3.15) we have extended spacetime $x^{\mu}=\frac{1}{\sqrt{2}}\left(\sigma^{\mu}\right)_{\alpha \dot{\beta}} x^{\alpha \dot{\beta}}$ by the three supplementary real four-vectors $y_{r}^{\mu}=\frac{1}{\sqrt{2}}\left(\sigma^{\mu}\right)_{\alpha \dot{\beta}} y_{r}^{\alpha \dot{\beta}}$. The parameter $c$ is real and $f$ is complex; $\rho$ and $\bar{\rho}$ are complex Lagrange multipliers that impose the spinorial mass shell constraints.

When $c=f=0, S^{(4)}$ describes the standard bispinorial Shirafuji model, with the pair of standard incidence relations

$$
\bar{\omega}^{\dot{\alpha} i}=\pi_{\beta}^{i} x^{\beta \dot{\alpha}}, \quad \omega_{i}^{\alpha}=x^{\alpha \dot{\beta}} \bar{\pi}_{\dot{\beta} i} .
$$

The reality of the spacetime coordinates $x^{\mu}$ implies, after multiplying the first equation above on the right side by $A_{i}{ }^{j} \bar{\pi}_{\dot{\alpha} j}$ and the second one on the left side by $\pi_{\alpha}^{j} A^{j i}$, the constraint

$$
\pi_{\alpha}^{j} A_{j}{ }^{i} \omega_{i}^{\alpha}-\bar{\omega}^{\dot{\alpha} i} A_{i}^{j} \bar{\pi}_{\dot{\alpha} j}=0
$$


which depends on the arbitrary hermitian $2 \times 2$ matrix $A_{i}{ }^{j}$, i.e. $\left(A_{i}{ }^{j}\right)^{\dagger}=A_{j}{ }^{i}$. Using the $\sigma^{a}$ basis of $2 \times 2$ hermitian matrices (appendix A), eq. (3.17) gives the following four linearly independent constraints $(a=(0 ; r)=(0 ; 1,2,3))$

$$
S_{a} \equiv-\frac{i}{2}\left[\pi_{\alpha}^{j}\left(\sigma_{a}\right)_{j}{ }^{i} \omega_{i}^{\alpha}-\bar{\omega}^{\dot{\alpha} i}\left(\sigma_{a}\right)_{i}{ }^{j} \bar{\pi}_{\dot{\alpha} j}\right]=0
$$

which can also be expressed by the four conformal scalar products of the twistors $Z_{A}^{i}, \bar{Z}_{A}^{i}$,

$$
S_{a} \equiv-\frac{i}{2} Z^{A i}\left(\sigma_{a}\right)_{i}{ }^{j} \bar{Z}_{A j}=0 .
$$

If relation (3.19) is valid, we see that the twistors generated by the incidence relation (3.16) are null twistors located on the null plane. The four constraints (3.19) and two spinorial mass constraints (1.10) provide four first class constraints and two of second class (see also [43]), i.e. if $c=f=0$ we obtain $16-2 \times 4-2=6$ physical degrees of freedom describing the physical phase space of massive spinless particle.

In the general case when $c \neq 0$ and $f \neq 0$ the proper generalization of the incidence relations is the following

$$
\begin{aligned}
\bar{\omega}^{\dot{\alpha} i} & =\pi_{\beta}^{i} x^{\beta \dot{\alpha}}+c \pi_{\beta}^{j}\left(\sigma^{r}\right)_{j}{ }^{i} y_{r}^{\beta \dot{\alpha}}+\bar{f} \bar{y}^{\dot{\alpha} i}, \\
\omega_{i}^{\alpha} & =x^{\alpha \dot{\beta}} \bar{\pi}_{\dot{\beta} i}+c y_{r}^{\alpha \dot{\beta}}\left(\sigma^{r}\right)_{i}{ }_{i} \bar{\pi}_{\dot{\beta} j}+f y_{i}^{\alpha} .
\end{aligned}
$$

Repeating the derivation of the constraints (3.17), we obtain in place of the formulae (3.18) the following relations $(i, j=1,2 ; r=1,2,3)$ :

$$
\begin{aligned}
& S_{0}=-\frac{i}{2}\left(f \pi_{\alpha}^{i} y_{i}^{\alpha}-\bar{f} \bar{y}^{\dot{\alpha} i} \bar{\pi}_{\dot{\alpha} i}\right), \\
& S_{r}=c \epsilon_{r p q} y_{p}^{\alpha \dot{\beta}} u_{q \alpha \dot{\beta}}+\frac{i}{2}\left[f \pi_{\alpha}^{i}\left(\sigma_{r}\right)_{i}{ }^{j} y_{j}^{\alpha}-\bar{f} \bar{y}^{\dot{\alpha} i}\left(\sigma_{r}\right)_{i}{ }^{j} \bar{\pi}_{\dot{\alpha} j}\right],
\end{aligned}
$$

where $u_{r \alpha \dot{\beta}}$ is given by formula (3.3). The independence of the first expression in (3.21) on the parameter $c$ follows from the reality of the four-vector coordinates $y_{a}^{\alpha \dot{\beta}} \sim\left(x^{\mu}, y_{r}^{\mu}\right)$.

To describe the phase space structure of the model (3.14) we calculate the momenta $p_{\alpha \dot{\beta}}^{a}, p_{(\pi)_{i}}^{\alpha}, p_{(\pi)}^{\dot{\alpha} i}, p_{(y) \alpha}^{i}, p_{(y) \dot{\alpha} i}$ conjugate to $y_{a}^{\alpha \dot{\beta}}, \pi_{\alpha}^{i}, \bar{\pi}_{\dot{\alpha} i}, y_{i}^{\alpha}, \bar{y}^{\dot{\alpha} i}$. This leads to the constraints (we set $c=f=1$ for simplicity)

$$
\begin{aligned}
T_{\alpha \dot{\beta}}^{a} & =p_{\alpha \dot{\beta}}^{a}-u_{\alpha \dot{\beta}}^{a} \approx 0, & & \\
G_{\alpha}^{i} & =p_{(y) \alpha} i \pi_{\alpha}^{i} \approx 0, & & \bar{G}_{\dot{\alpha} i}=\bar{p}_{(y) \dot{\alpha} i}-\bar{\pi}_{\dot{\alpha} i} \approx 0, \\
F_{i}^{\alpha} & =p_{(\pi) i}{ }_{i}^{\alpha} \approx 0, & & \bar{F}^{\dot{\alpha} i}=\bar{p}_{(\pi)}{ }^{\dot{\alpha} i} \approx 0 .
\end{aligned}
$$

The remaining two (mass) constraints are given by (1.10).

The constraints (3.23) and (3.24) are of second class. Introducing the corresponding Dirac brackets $\{A, B\} \rightarrow\{A, B\}_{*}$ and eliminating by (3.23) the momenta $p_{(y) \alpha}, \bar{p}_{(y) \dot{\alpha} i}$ we get the following set of Dirac brackets taking the canonical form

$$
\left\{y_{a}^{\gamma \dot{\delta}}, p_{\alpha \dot{\beta}}^{b}\right\}_{*}=\delta_{a}^{b} \delta_{\alpha}^{\gamma} \delta_{\dot{\beta}}^{\dot{\delta}}, \quad\left\{y_{i}^{\alpha}, \pi_{\beta}^{j}\right\}_{*}=\delta_{i}^{j} \delta_{\beta}^{\alpha}, \quad\left\{\bar{y}^{\dot{\alpha} i}, \bar{\pi}_{\dot{\beta} j}\right\}_{*}=\delta_{j}^{i} \delta_{\dot{\beta}}^{\dot{\alpha}}
$$


The constraints (1.10) and (3.22) are first class. By quantizing the brackets (3.25) and introducing the realization

$$
\hat{y}_{a}^{\alpha \dot{\beta}}=y_{a}^{\alpha \dot{\beta}}, \quad \hat{p}_{\alpha \dot{\beta}}^{a}=-i \frac{\partial}{\partial y_{a}^{\alpha \dot{\beta}}},
$$

we obtain the $D=4$ unfolded equation for the wave function $\Psi\left(y_{a}^{\alpha \dot{\beta}}, \pi_{\alpha}^{i}, \bar{\pi}_{\dot{\alpha} i}\right)$ :

$$
\left(i \frac{\partial}{\partial y_{a}^{\alpha \dot{\beta}}}+\pi_{\alpha}^{i}\left(\sigma^{a}\right)_{i}{ }^{j} \bar{\pi}_{\dot{\beta} j}\right) \Psi\left(y_{a}^{\alpha \dot{\beta}}, \pi_{\alpha}^{i}, \bar{\pi}_{\dot{\alpha} i}\right)=0 .
$$

The equation (3.27) has the solution $(a=0,1,2,3)$

$$
\Psi\left(y_{a}^{\alpha \dot{\beta}}, \pi_{\alpha}^{i}, \bar{\pi}_{\dot{\alpha} i}\right)=\exp \left\{i \pi_{\alpha}^{i}\left(\sigma^{a}\right)_{i}{ }^{j} \bar{\pi}_{\dot{\beta} j} y_{a}^{\alpha \dot{\beta}}\right\} \psi\left(\pi_{\alpha}^{i}, \bar{\pi}_{\dot{\alpha} i}\right),
$$

where the reduced wave functions $\psi(\pi, \bar{\pi})$ depend on complex $D=4$ spinorial momenta satisfying the mass constraints in (1.10). For the general model $(3.15)(c \neq 0, f \neq 0)$ it follows from (3.21) that all four variables $S^{a}$ are dynamical and that the reduced wave function $\psi(\pi, \bar{\pi})$ does not satisfy any further constraints besides (1.10).

The spinors $e_{\alpha}^{i}=(M)^{-\frac{1}{2}} \pi_{\alpha}^{i}\left(\bar{e}_{\dot{\alpha} i}=(\bar{M})^{-\frac{1}{2}} \bar{\pi}_{\dot{\alpha} i}\right)$ define a complex-holomorphic (complex anti-holomorphic) spinorial $\mathrm{SL}(2 ; \mathbb{C})$ Lorentz frame $(\mathrm{SL}(2 ; \mathbb{C})$ spinorial harmonics),

$$
e_{\alpha}^{i} e^{\alpha j}=\epsilon^{i j}, \quad e_{\alpha}^{i} e_{\beta i}=\epsilon_{\alpha \beta} ; \quad \bar{e}_{\dot{\alpha}}^{i} e^{\dot{\alpha} j}=\epsilon^{i j}, \quad \bar{e}_{\dot{\alpha}}^{i} \bar{e}_{\dot{\beta} i}=\epsilon_{\dot{\alpha} \dot{\beta}},
$$

and the reduced wave function $\psi\left(\pi_{\alpha}^{i}, \bar{\pi}_{\dot{\alpha} i}\right)$ in (3.28) depends on an arbitrary element of the $\mathrm{SL}(2 ; \mathbb{C})$ group (see also [68]). The six unconstrained degrees of freedom can be described by the spinorial frame $e_{\alpha}^{i}(i=1,2, \alpha=1,2$, eq. (3.29)) or by the vectorial frame given by the four-vectors $e_{\mu}{ }^{a}(a=0,1,2,3 ; \mu=0,1,2,3)$ satisfying the orthonormality relation (3.8). In particular, following [53], one can incorporate five degrees of freedom into the pair of four-vectors

$$
p_{\mu}^{(0)}=m e_{\mu}^{(0)} \equiv p_{\mu}, \quad p_{\mu}^{(1)}=m e_{\mu}^{(1)} \equiv q_{\mu},
$$

satisfying the conditions

$$
p_{\mu} p^{\mu}=m^{2}, \quad q_{\mu} q^{\mu}=-m^{2}, \quad p_{\mu} q^{\mu}=0 .
$$

The four-vector $q_{\mu}$ parametrizes the sphere $\mathbb{S}^{2}$ in an arbitrary Lorentz frame. The remaining sixth degree of freedom can be described by the $\mathrm{SO}(2)$ angle $0 \leq \gamma<2 \pi$, defined by the third vector $r_{\mu}$

$$
p_{\mu}^{(2)}=m e_{\mu}^{(2)}=r_{\mu}, \quad r_{\mu} r^{\mu}=-m^{2}, \quad p_{\mu} r^{\mu}=q_{\mu} r^{\mu}=0 .
$$

In the rest frame, $p_{\mu}=(m, 0,0,0)$, the four-vector $r_{\mu}$ can be parametrized as

$$
r_{\mu}=(0,0, m \cos \gamma, m \sin \gamma) .
$$

Therefore, the reduced wave function (3.28) incorporating the mass constraints (1.10) can be parametrized as

$$
\left.\psi\left(\pi_{\alpha}^{i}, \bar{\pi}_{\dot{\alpha} i}\right) \equiv \hat{\psi}\left(p_{\mu}, \mathbb{S}^{2}, \mathbb{S}^{1}\right)\right|_{p^{2}=m^{2}},
$$


where $\mathbb{S}^{2}$ is described by $q_{\mu}$ and $\mathbb{S}^{1}$ parametrizes $r_{\mu}$ by eq. (3.33). To describe the $D=4$ integer spin states we may neglect the dependence on the $\mathbb{S}^{1}$ parameter; however for halfinteger spins the dependence on the angle $\gamma$ becomes necessary (see e.g. [53]).

Let us consider now the model (3.15) for $f=0, c \neq 0$, i.e. without kinetic spinorial terms introduced by Vasiliev [4] in order to obtain the unfolded equations (see (3.27)). We shall follow the arguments given for $D=3$ in the last part of section 2.2. When $f=0$ one obtains the constraints (1.10), (3.22), (3.24) but not the constraints (3.23). Using (3.8), we introduce for (3.22) and (3.24) the equivalent set of sixteen real and four pairs of complex-conjugated constraints $(a, b=0,1,2,3)$

$$
\begin{aligned}
T_{b}^{a} & =T_{\alpha \dot{\beta}}^{a} u_{b}^{\alpha \dot{\beta}}=p_{\alpha \dot{\beta}}^{a} u_{b}^{\alpha \dot{\beta}}-m^{2} \delta_{b}^{a} \approx 0, \\
F_{a} & =\frac{1}{2} \pi_{\alpha}^{i}\left(\sigma_{a}\right)_{i}{ }^{j} p_{(\pi) j}^{\alpha} \approx 0, \quad \bar{F}_{a}=\frac{1}{2} p_{(\bar{\pi})}^{\dot{\alpha} i}\left(\sigma_{a}\right)_{i}{ }^{j} \bar{\pi}_{\dot{\alpha} j} \approx 0 .
\end{aligned}
$$

The nonvanishing Dirac brackets (see (3.25)) of the constraints (3.35), (3.36) and (1.10) are $\left(F_{a}=\left(F_{0}, F_{r}\right)\right)$

$$
\begin{array}{ll}
\left\{F_{q}, F_{r}\right\}_{*}=-i \epsilon_{q r s} F_{s}, & \left\{\bar{F}_{q}, \bar{F}_{r}\right\}_{*}=i \epsilon_{q r s} \bar{F}_{s}, \\
\left\{F_{0}, T_{b}^{a}\right\}_{*}=-\frac{1}{2} T_{b}^{a}-\frac{1}{2} \delta_{b}^{a} m^{2}, & \left\{\bar{F}_{0}, T_{b}^{a}\right\}_{*}=-\frac{1}{2} T_{b}^{a}-\frac{1}{2} \delta_{b}^{a} m^{2}, \\
\left\{F_{r}, T_{0}^{a}\right\}_{*}=-\frac{1}{2} T_{r}^{a}-\frac{1}{2} \delta_{r}^{a} m^{2}, & \left\{\bar{F}_{r}, T_{0}^{b}\right\}_{*}=-\frac{1}{2} T_{r}^{b}-\frac{1}{2} \delta_{r}^{b} m^{2}, \\
\left\{F_{r}, T_{q}^{a}\right\}_{*}=-\frac{i}{2} \epsilon_{r q s} T_{s}^{a}-\frac{i}{2} \epsilon_{r q s} \delta_{s}^{a} m^{2}-\frac{1}{2} T_{0}^{a} \delta_{r q}-\frac{1}{2} m^{2} \delta_{0}^{a} \delta_{r q}, & \left\{\bar{F}_{r}, T_{q}^{a}\right\}_{*}=\frac{i}{2} \epsilon_{r q s} T_{s}^{a}+\frac{i}{2} \epsilon_{r q s} \delta_{s}^{a} m^{2}-\frac{1}{2} T_{0}^{a} \delta_{r q}-\frac{1}{2} m^{2} \delta_{0}^{a} \delta_{r q}, \\
\left\{F_{0}, \mathcal{M}\right\}_{*}=-\mathcal{M}+2 M, & \left\{\bar{F}_{0}, \overline{\mathcal{M}}\right\}_{*}=-\overline{\mathcal{M}}+2 \bar{M},
\end{array}
$$

where $q, r, s=1,2,3$. We see that the 8 real constraints $F_{a}, \bar{F}_{a}$ provide a partial gauge fixing of the $18=16+2$ gauge transformations, which in the case $f \neq 0$ are generated by the $18=16+2$ first class constraints $T_{b}^{a}, \mathcal{M}, \overline{\mathcal{M}}$. One can calculate that if $c \neq 0$ the variants $f \neq 0$ and $f=0$ of the model (3.15) have the same number of twelve real physical (nongauge) degrees of freedom but different number (18 for $f \neq 0$ and 10 for $f=0$ ) of local (i.e. $\tau$-dependent) gauge parameters.

We add that for a $D=4$ particle of mass $m$ and fixed spin $s$ the physical phase space has eight degrees of freedom, with the spin degrees represented e.g. by the coordinates on the sphere $\mathbb{S}^{2}[53,69]$. In such a theory the relation (3.13) that determines the fixed spin value $s$ is a first class constraint. If this constraint is removed, the resulting theory with arbitrary spin $s$ has then ten degrees of freedom. It will be shown in section 3.4 that the wave function solving the model (3.15) describes twelve degrees of freedom due to the multiplicity that is associated with each value of the different spins. We shall reduce the twelve degrees of freedom to ten, as required by a HS theory with nondegenerate spin spectrum, by imposing an harmonicity constraint (see (3.81) below) on the wave function.

\section{3 $D=4$ bitwistorial description of HS massive multiplets}

Following the procedure in section 2 for $D=3$, we now express the action (3.15) just in terms of a pair of $D=4$ twistor coordinates (eq. (3.1)) by postulating the incidence 
relations (3.20). With $f \neq 0$ ( $c$ may be arbitrary) this leads to the following two-twistorial action with two complex-conjugated Lagrange multipliers $\mu, \bar{\mu}$

$$
\tilde{S}^{(4)}=\int d \tau\left[\pi_{\alpha}^{i} \dot{\omega}_{i}^{\alpha}+\mu\left(\pi_{\alpha}^{i} \pi_{i}^{\alpha}+2 M\right)+\text { h.c. }\right] .
$$

The model (3.42) contains only two complex-conjugated spinorial mass constraints (1.10). When $f=0$ and $c \neq 0$, as it follows from formulae (3.21), one still has to to impose one additional constraint via a Lagrange multiplier

$$
S_{0}=-\frac{i}{2} \bar{Z}_{A i} Z^{A i} \approx 0 .
$$

In order to find the first and second class constraints we use the canonical PB that follow from the $\tilde{S}^{(4)}$ action (3.42)

$$
\left\{\bar{Z}_{A i}, Z^{B j}\right\}=\delta_{i}^{j} \delta_{A}^{B}
$$

One can check that $\left\{\mathcal{M}, S^{0}\right\} \neq 0,\left\{\overline{\mathcal{M}}, S^{0}\right\} \neq 0$. Further, we replace the two complexconjugated constraints $\mathcal{M}, \overline{\mathcal{M}}$ by a pair of real constraints

$$
\begin{aligned}
& \phi_{1}=\frac{1}{2}(\mathcal{M}+\overline{\mathcal{M}})=\frac{1}{2}\left(\pi_{\alpha}^{i} \pi_{i}^{\alpha}+\text { h.c. }\right)-M_{1}=0, \\
& \phi_{2}=\frac{i}{2}(\mathcal{M}-\overline{\mathcal{M}})=\frac{i}{2}\left(\pi_{\alpha}^{i} \pi_{i}^{\alpha} \text { - h.c. }\right)-M_{2}=0,
\end{aligned}
$$

where $M=M_{1}+i M_{2}$. The $\mathrm{PB}$ of the constraints $\left(S_{0}, \phi_{1}, \phi_{2}\right)$ are

$$
\begin{aligned}
& \left\{S_{0}, \phi_{1}\right\}=\phi_{2}+M_{2}, \\
& \left\{S_{0}, \phi_{2}\right\}=-\phi_{1}-M_{1}, \\
& \left\{\phi_{1}, \phi_{2}\right\}=0 .
\end{aligned}
$$

The PBs in eq. (3.46) show that the generators $S_{0}, \phi_{1}^{\prime}=\phi_{1}+M_{1}, \phi_{2}^{\prime}=\phi_{2}+M_{2}$ describe an $E(2)$ algebra, $\left\{S_{0}, \phi_{1}^{\prime}\right\}=\phi_{2}^{\prime},\left\{S_{0}, \phi_{2}^{\prime}\right\}=-\phi_{1}^{\prime},\left\{\phi_{1}^{\prime}, \phi_{2}^{\prime}\right\}=0$.

The shifts $\phi_{1}^{\prime}, \phi_{2}^{\prime} \rightarrow \phi_{1}, \phi_{2}$ of the generators of the translation sector of $E(2)$ may be considered as producing spontaneously broken symmetries. Indeed, after quantization of PB (3.46) one can consider that the action of the $E(2)$ generators $\left(\hat{S}_{0}, \hat{\phi}_{1}^{\prime}, \hat{\phi}_{2}^{\prime}\right)$ annihilates the vacuum $|0\rangle$. Then, the quantized relations (3.46) are consistent only if $\hat{S}_{0}|0\rangle=0$, $\hat{\phi}_{1,2}^{\prime}|0\rangle=0 \Rightarrow \hat{\phi}_{1,2}|0\rangle=M_{1,2}|0\rangle \neq 0$. This means that if we look at $\hat{\phi}_{1}, \hat{\phi}_{2}$ as generating the two translational symmetries of $E(2)$ these have to be spontaneously broken. ${ }^{9}$ Similarly, if we introduce another choice of generators

$$
\widetilde{\phi}_{1}=M_{1} \phi_{1}+M_{2} \phi_{2}, \quad \widetilde{\phi}_{2}=M_{2} \phi_{1}-M_{1} \phi_{2},
$$

\footnotetext{
${ }^{9}$ We recall that the symmetry associated with a Lie algebra generator $\hat{X}$ is spontaneously broken if $\hat{X}|0\rangle \neq 0$ [70]. The phenomenon above described is that if $\hat{\phi}_{1}, \hat{\phi}_{2}$ are considered as translation generators, then we cannot longer ignore that the true algebra is larger and that, in it, the constants determine a central subalgebra. Taking a basis that it is not a subalgebra led to the symmetry breaking above.
} 
the PB (3.46) will be rewritten as representing $E(2)$ algebra broken spontaneously only in one translational direction generated by $\widetilde{\phi}_{1}$

$$
\begin{aligned}
& \left\{S_{0}, \widetilde{\phi}_{1}\right\}=\widetilde{\phi}_{2}, \\
& \left\{S_{0}, \widetilde{\phi}_{2}\right\}=-\widetilde{\phi}_{1}-m^{2}, \\
& \left\{\widetilde{\phi}_{1}, \widetilde{\phi}_{2}\right\}=0,
\end{aligned}
$$

where $m^{2}=|M|^{2}=M_{1}^{2}+M_{2}^{2}$. We see from (3.48) that the constraint $\widetilde{\phi}_{1}$ is of first class, and $\widetilde{\phi}_{2}, S_{0}$ form a pair of second class constraints.

It turns out nevertheless that the number of physical phase space degrees of freedom is the same and equal to twelve, irrespectively of the value of the parameter $f$. In fact,

1. if $f \neq 0$ we have two first class constraints (1.10), i.e. in 16-dimensional two-twistor phase space the number of degrees of freedom is $16-2 \times 2=12$.

2. if $f=0$ and $c \neq 0$ we get three constraints satisfying the PBs (3.48), one first class and two second class. The count of degrees of freedom is the same: $16-1 \times 2-2 \times 1=12$.

3. If $f=0$ and $c=0$ we obtain the model of massive spinless particle (see formulae (3.15)-(3.19)), with six-dimensional physical phase space.

In the fist two cases we obtain the twelve dimensions of physical phase space by doubling the number of independent coordinates that parametrize the six-dimensional manifold $\operatorname{SL}(2 ; \mathbb{C})$; in accordance with (3.34), the reduced wave function is defined on this manifold.

To relate more closely our description with the spin degrees of freedom, let us recall the Lorentz-invariant spin variables $S_{r}$ defined by eq. (3.19). Using the PB relations in (3.44), one can show that the bilinears $S_{r}$ satisfy the $s o(3) \simeq s u(2) \mathrm{PB}$ algebra $(q, p, r=1,2,3)$

$$
\left\{S_{q}, S_{p}\right\}=\epsilon_{q p r} S_{r}
$$

In particular, if $S_{r} \approx 0 \Rightarrow W_{\alpha \dot{\beta}} \approx 0$ (see (3.11)), i.e. the spin is equal to zero. In our twistorial model $S_{r} \neq 0$ (see (3.21)) and after quantization $\left(S_{r} \rightarrow \hat{S}_{r}\right)$ ) we obtain from (3.49) the so(3) algebra of Lorentz-invariant spin generators $\hat{S}_{r}$

$$
\left[\hat{S}_{q}, \hat{S}_{p}\right]=i \epsilon_{q p r} \hat{S}_{r}
$$

The mass shell constraints, after using the bitwistor formula (3.4) for the four-momentum, provide the generalized Dirac equation with complex mass $M$ and four-components complex Dirac spinors

$$
\pi^{\beta i} p_{\alpha \dot{\beta}}=M \bar{\pi}_{\dot{\alpha}}^{i}, \quad p_{\alpha \dot{\beta}} \bar{\pi}^{\dot{\beta} i}=\bar{M} \pi_{\alpha}^{i} .
$$

Further, in our two-twistor framework we obtain as well the generalization of eqs. (3.51) for the set of three auxiliary fourmomenta $(r=1,2,3)$,

$$
\pi^{\alpha i} p_{\alpha \dot{\beta}}^{r}=M \bar{\pi}_{\dot{\beta}}^{j}\left(\sigma^{r}\right)_{j}{ }^{i}, \quad p_{\alpha \dot{\beta}}^{r} \bar{\pi}^{\dot{\beta} j}=\bar{M} \pi_{\alpha}^{i}\left(\sigma^{r}\right)_{i}{ }^{j}
$$


To replace the complex value $M=\frac{1}{\sqrt{2}} e^{i \varphi} m$ by a real one $m$ let us observe that the action (3.15) is invariant under the following global phase transformations

$$
\begin{aligned}
\pi_{\alpha}^{\prime i} & =e^{i \varphi / 2} \pi_{\alpha}^{i}, & \bar{\pi}_{\dot{\alpha} i}^{\prime} & =e^{-i \varphi / 2} \bar{\pi}_{\dot{\alpha} i}, \\
f^{\prime} & =e^{-i \varphi / 2} f, & \bar{f}^{\prime} & =e^{i \varphi / 2} \bar{f} ; \quad \rho^{\prime}=e^{-i \varphi} \rho, \quad \bar{\rho}^{\prime}=e^{i \varphi} \bar{\rho},
\end{aligned}
$$

where $e^{2 i \varphi}=M / \bar{M}$. The $D=4$ mass constraints (1.10) are expressed in terms of $\pi_{\alpha}^{\prime i}, \bar{\pi}_{\dot{\alpha} i}^{\prime}$ by (cf. eq. (2.6) for $D=3$ )

$$
\pi_{\alpha}^{\prime i} \pi_{i}^{\prime \alpha}+\sqrt{2} m=0, \quad \bar{\pi}_{\dot{\alpha}}^{\prime i} \bar{\pi}_{i}^{\prime \dot{\alpha}}+\sqrt{2} m=0 .
$$

For the Weyl spinors $\pi_{\alpha}^{\prime i}, \bar{\pi}_{\dot{\alpha} i}^{\prime}$ we get the equations (3.51), (3.52) with $M$ replaced by $m$. The transformations $(3.53)$ do not affect the $\mathrm{SL}(2 ; \mathbb{C})$ part of the variables $\pi_{\alpha}^{i}$ (see next section) because they change only the determinant of the $2 \times 2$ matrix $\pi_{\alpha}^{i}$, which is parametrized by the coset $G L(2 ; \mathbb{C}) / \mathrm{SL}(2 ; \mathbb{C}) \simeq G L(1 ; \mathbb{C})$, parametrized by an arbitrary complex mass parameter.

\section{4 $D=4$ bitwistor wave function of HS massive multiplet}

Our $D=4$ dynamical bitwistorial system is described by twistorial coordinates see (3.1)) in terms of the variables $\pi_{\alpha}^{j}, \bar{\pi}_{\dot{\alpha} k}, \omega_{k}^{\alpha}, \bar{\omega}^{\dot{\alpha} i}$ endowed with the canonical PBs

$$
\left\{\omega_{i}^{\alpha}, \pi_{\beta}^{j}\right\}=\delta_{\beta}^{\alpha} \delta_{i}^{j}, \quad\left\{\bar{\omega}^{\dot{\alpha} i}, \bar{\pi}_{\dot{\beta} j}\right\}=\delta_{\dot{\beta}}^{\dot{\alpha}} \delta_{j}^{i},
$$

constrained by the mass constraints $\mathcal{M}, \overline{\mathcal{M}}$ (eqs. (1.10)). Further we shall assume $f=0$ and $c \neq 0$. In such a case we should add the constraint (3.43)

$$
V=-2 S_{0}=i\left(\pi_{\alpha}^{i} \bar{\omega}_{i}^{\alpha}-\bar{\pi}_{\dot{\alpha} i} \omega^{\dot{\alpha} i}\right) \approx 0
$$

with nonvanishing PBs

$$
\{V, \mathcal{M}\}=2 i \mathcal{M}+4 i M, \quad\{V, \overline{\mathcal{M}}\}=-2 i \overline{\mathcal{M}}-4 i \bar{M} .
$$

The constraints $\mathcal{M}, \overline{\mathcal{M}}$ can be equivalently described by

$$
F_{1}=\bar{M} \mathcal{M}+M \overline{\mathcal{M}}, \quad F_{2}=i(\bar{M} \mathcal{M}-M \overline{\mathcal{M}}),
$$

One can check easily that the constraints $V$ and $F_{2}$ are second class. For the local gauge transformations generated by the constraint $F_{1}$ we introduce the gauge fixing condition

$$
G=\pi_{\alpha}^{i} \bar{\omega}_{i}^{\alpha}+\bar{\pi}_{\dot{\alpha} i} \omega^{\dot{\alpha} i} \approx 0,
$$

described by the generator of scale transformations (dilatations) for twistorial variables. Further, using (3.55), (1.10) and (3.59) one obtains

$$
\{G, \mathcal{M}\}=2 \mathcal{M}+4 M, \quad\{G, \overline{\mathcal{M}}\}=2 \overline{\mathcal{M}}+4 \bar{M} .
$$


The $\mathrm{PB}$ of the constraints $V, G, F_{1}$ and $F_{2}$ are

$$
\begin{array}{llrl}
\left\{G, F_{1}\right\} & =2 F_{1}+8 M \bar{M}, & \left\{G, F_{2}\right\} & =2 F_{2}, \\
& \left\{V, F_{1}\right\}=2 F_{2}, & \left\{V, F_{2}\right\} & =-2 F_{1}-8 M \bar{M} .
\end{array}
$$

Then, the Dirac brackets (DB) that account for the four second class constraints (3.61) are defined by the formula

$$
\begin{aligned}
\{A, B\}_{*}= & \{A, B\}+ \\
& \frac{1}{8 M M}\left[\{A, G\}\left\{F_{1}, B\right\}-\left\{A, F_{1}\right\}\{G, B\}-\{A, V\}\left\{F_{2}, B\right\}+\left\{A, F_{2}\right\}\{V, B\}\right] .
\end{aligned}
$$

This gives for the twistor components the DBs

$$
\begin{aligned}
\left\{\pi_{\alpha}^{k}, \pi_{\beta}^{j}\right\}_{*} & =\left\{\bar{\pi}_{\dot{\alpha} k}, \bar{\pi}_{\dot{\beta} j}\right\}_{*}=\left\{\pi_{\alpha}^{k}, \bar{\pi}_{\dot{\beta} j}\right\}_{*}= & 0, \\
\left\{\omega_{k}^{\alpha}, \pi_{\beta}^{j}\right\}_{*} & =\delta_{\beta}^{\alpha} \delta_{k}^{j}+\frac{1}{2 M} \pi_{k}^{\alpha} \pi_{\beta}^{j}, & \left\{\bar{\omega}^{\dot{\alpha} k}, \bar{\pi}_{\dot{\beta} j}\right\}_{*}=\delta_{\dot{\beta}}^{\dot{\alpha}} \delta_{j}^{k}-\frac{1}{2 M} \bar{\pi}^{\dot{\alpha} k} \bar{\pi}_{\dot{\beta} j}, \\
\left\{\bar{\omega}^{\dot{\alpha} k}, \pi_{\beta}^{j}\right\}_{*} & =0, & \left\{\omega_{k}^{\alpha}, \bar{\pi}_{\dot{\beta} j}\right\}_{*}=0, \\
\left\{\omega_{k}^{\alpha}, \omega_{j}^{\beta}\right\}_{*} & =-\frac{1}{M}\left(\pi_{k}^{\alpha} \bar{\omega}_{j}^{\beta}-\pi_{j}^{\beta} \bar{\omega}_{k}^{\alpha}\right), & \left\{\bar{\omega}^{\dot{\alpha} k}, \bar{\omega}^{\dot{\beta} j}\right\}_{*}=\frac{1}{M}\left(\bar{\pi}^{\dot{\alpha} k} \omega^{\dot{\beta} j}-\bar{\pi}^{\dot{\beta} j} \omega^{\dot{\alpha} k}\right), \\
\left\{\omega_{k}^{\alpha}, \bar{\omega}^{\dot{\beta} j}\right\}_{*} & =0 . &
\end{aligned}
$$

Below we will consider the $(\pi, \bar{\pi})$-realization of the quantized version of the DB algebra (3.63)-(3.67). In such a realization, after using the ordering with $\pi$ 's at the left and $\omega$ 's at the right, we obtain $\hat{\pi}_{\alpha}^{k}=\pi_{\alpha}^{k}, \hat{\bar{\pi}}_{\dot{\alpha} k}=\bar{\pi}_{\dot{\alpha} k}$ and

$$
\hat{\omega}_{k}^{\alpha}=i \frac{\partial}{\partial \pi_{\alpha}^{k}}+\frac{i}{2 M} \pi_{k}^{\alpha} \pi_{\beta}^{j} \frac{\partial}{\partial \pi_{\beta}^{j}}, \quad \hat{\bar{\omega}}^{\dot{\alpha} k}=i \frac{\partial}{\partial \bar{\pi}_{\dot{\alpha} k}}-\frac{i}{2 \bar{M}} \bar{\pi}^{\dot{\alpha} k} \bar{\pi}_{\dot{\beta} j} \frac{\partial}{\partial \bar{\pi}_{\dot{\beta} j}} .
$$

one checks that in the presence of $D=4$ mass constraints (1.10) the constraints (3.56), (3.59) are satisfied in the strong sense: $\hat{\pi}_{\alpha}^{k} \hat{\omega}_{k}^{\alpha} \equiv 0, \hat{\bar{\pi}}_{\dot{\alpha} k} \hat{\bar{\omega}}^{\dot{\alpha} k} \equiv 0$.

Taking into account the expressions (3.68) we obtain the quantum counterparts of the quantities (3.12) as the spin operators

$$
\hat{S}_{r}=\frac{1}{2}\left(\pi_{\alpha}^{i} \frac{\partial}{\partial \pi_{\alpha}^{k}}-\bar{\pi}_{\dot{\alpha} k} \frac{\partial}{\partial \bar{\pi}_{\dot{\alpha} i}}\right)\left(\sigma_{r}\right)_{i}{ }^{k} .
$$

Using (3.13), the square of the Pauli-Lubański vector becomes $\hat{W}^{\mu} \hat{W}_{\mu}=-m^{2} \hat{S}^{r} \hat{S}^{r}$, which will be used later to define spin states.

Thus, the twistorial wave function is defined on the space parametrized by $\pi_{\alpha}^{i}, \bar{\pi}_{\dot{\alpha} i}$ which satisfy the constraints $\mathcal{M}, \overline{\mathcal{M}}$ (eq. (1.10)), and the matrix

$$
g_{\alpha}{ }^{i}=M^{-1 / 2} \pi_{\alpha}^{i}
$$

defines the $\operatorname{SL}(2, \mathbb{C})$ group manifold. Thus, the twistorial wave function is defined on $\operatorname{SL}(2, \mathbb{C})$ parametrized by $\pi_{\alpha}^{i}$, so that $\Psi=\Psi\left(\pi_{\alpha}^{i}, \bar{\pi}_{\dot{\alpha} i}\right)$. One can use the well known decomposition of $\operatorname{SL}(2, \mathbb{C})$ elements

$$
g=h v, \quad g_{\alpha}{ }^{i}=h_{\alpha}{ }^{\mathrm{k}} v_{\mathrm{k}}{ }^{i},
$$


in terms of the product of an hermitian matrix $h=h^{\dagger}$ with unit determinant and an $\mathrm{SU}(2)$ matrix $v, v^{\dagger} v=1$ (in the above formulae, the $v_{\mathrm{k}}{ }^{i}$ play the role of $\psi$ in (2.41) for $D=3)$. The three parameters of the matrix $h$ describe four-momenta on the mass shell, and the three parameters of the matrix $v$ correspond to the spin algebra (3.50). The matrix $h$ parametrizes the coset $\mathrm{SL}(2, \mathbb{C}) / \mathrm{SU}(2)$ which defines the three-dimensional mass hyperboloid for timelike four-momenta which does not depend on the $v_{\mathrm{k}}{ }^{i}$ variables (as in $D=3$ eqs. (2.48) do not depend on $\psi$ ). So, the definition (3.4) can be rewritten as follows

$$
p_{\alpha \dot{\beta}}=h_{\alpha}{ }^{\mathrm{i}} \bar{h}_{\dot{\beta} \dot{\mathrm{i}}}
$$

where $\bar{h}_{\dot{\alpha} \mathrm{i}}=\left(h_{\alpha}{ }^{\mathrm{i}}\right)^{*}$ and $\alpha=1,2$ and $\mathrm{i}=1,2$.

The unitary matrix $v$ parametrizes $\mathbb{S}^{3} \sim \mathrm{SU}(2)$ and is linked with the spin degrees of a massive particle. In particular, the operators (3.69) expressed by the variables (3.71) take the form

$$
\hat{S}_{r}=\frac{1}{2}\left(\sigma_{r}\right)_{j}^{k} v_{\mathrm{i}}{ }^{j} \frac{\partial}{\partial v_{\mathrm{i}}^{k}} .
$$

We can consider the variables $v_{\mathrm{i}}{ }^{k}$ as the harmonic variables that were introduced earlier to describe $N=2$ superfield formulations (see, for example, [71]). In particular, it is useful to introduce the notation

$$
v_{\mathrm{i}}^{k}=\left(v_{\mathrm{i}}^{1}, v_{\mathrm{i}}^{2}\right)=\left(v_{\mathrm{i}}^{+}, v_{\mathrm{i}}^{-}\right), \quad v^{+\mathrm{i}} v_{\mathrm{i}}^{-}=1, \quad\left(v_{\mathrm{i}}^{ \pm}\right)^{*}=\mp v^{\mp \mathrm{i}} .
$$

Then, the operators (3.73) take the form

$$
D^{0} \equiv 2 \hat{S}_{3}=v_{i}^{+} \frac{\partial}{\partial v_{i}^{+}}-v_{i}^{-} \frac{\partial}{\partial v_{i}^{-}}, \quad D^{ \pm \pm} \equiv \hat{S}_{1} \pm i \hat{S}_{2}=v_{i}^{ \pm} \frac{\partial}{\partial v_{i}^{\mp}},
$$

and the square of the Pauli-Lubański vector is given by the formula

$$
\hat{W}^{\mu} \hat{W}_{\mu}=-\frac{m^{2}}{4}\left[\left(D^{0}\right)^{2}+2\left\{D^{++}, D^{--}\right\}\right] .
$$

Since the variables $v_{i}^{ \pm}$parametrize a compact space, the general wave function on $\mathrm{SL}(2, \mathbb{C})$ has the following harmonic expansion (we use the $\mathrm{SU}(2)$-covariant expansion from [71])

$$
\Psi\left(h_{\alpha}{ }^{i}, v_{\mathbf{i}}{ }^{k}\right)=\sum_{K, N=0}^{\infty} v_{\left(\mathbf{i}_{1}\right.}^{+} \ldots v_{\mathbf{i}_{N}}^{+} v_{\mathbf{j}_{1}}^{-} \ldots v_{\left.\mathbf{j}_{K}\right)}^{-} f^{\mathbf{i}_{1} \ldots \dot{i}_{N} \mathrm{j}_{1} \ldots \mathbf{j}_{K}}(h),
$$

where the coefficient fields $f^{\mathrm{i}_{1} \ldots \mathrm{i}_{N} \mathrm{j}_{1} \ldots \mathrm{j}_{K}}(h)=f^{\left(\mathrm{i}_{1} \ldots \mathrm{i}_{N} \mathrm{j}_{1} \ldots \mathrm{j}_{K}\right)}(h)$ are symmetric with respect to all indices because the antisymmetric contributions involving factors in $v^{+}$and $v^{-}$ disappear due to the formula

$$
v_{\mathrm{i}}^{+} v_{\mathrm{j}}^{-}-v_{\mathrm{j}}^{+} v_{\mathrm{i}}^{-}=\epsilon_{\mathrm{ij}},
$$

which follows from the second expression in the definition of harmonic variables (3.74). These coefficient fields depend on the on-shell four-momenta due to (3.72), $f^{\mathbf{i}_{1} \ldots \mathbf{i}_{N} \mathrm{j}_{1} \ldots \mathrm{j}_{K}}(h)=f^{\mathrm{i}_{1} \ldots \mathbf{i}_{N} \mathrm{j}_{1} \ldots \mathrm{j}_{K}}\left(p_{\mu}\right)$. Such functions defined on the mass hyperboloid can be expanded into $\mathrm{SL}(2 ; \mathbb{C})$ irreducible representations belonging to the principal series of the first kind [72]. 
Each monomial of the variables $v_{i}^{ \pm}$in the expansion (3.77) is an eigenvector of the Casimir operator (3.76):

$$
\begin{aligned}
& \hat{W}^{\mu} \hat{W}_{\mu} v_{\mathbf{i}_{1}}^{+} \ldots v_{\mathbf{i}_{N}}^{+} v_{\mathrm{j}_{1}}^{-} \ldots v_{\mathbf{j}_{K}}^{-} f^{\mathbf{i}_{1} \ldots \mathbf{i}_{N} \mathrm{j}_{1} \ldots \mathrm{j}_{K}}= \\
& \quad-m^{2} s(s+1) v_{\mathbf{i}_{1}}^{+} \ldots v_{\mathbf{i}_{N}}^{+} v_{\mathbf{j}_{1}}^{-} \ldots v_{j_{K}}^{-} f^{\mathbf{i}_{1} \ldots \mathbf{i}_{N} j_{1} \ldots j_{K}}
\end{aligned}
$$

where $s=\frac{N+K}{2}$. So, the expression (3.77) is in fact the general expansion into arbitrary spin states. By means of the nonsingular transformation $v \rightarrow g=h v$, i.e. $v_{i}^{ \pm} \rightarrow \pi_{\alpha}^{ \pm}$or $v_{i}^{\mp} \rightarrow \bar{\pi}_{\dot{\alpha}}^{ \pm}$where

$$
\left(\pi_{\alpha}^{+}, \pi_{\alpha}^{-}\right)=\left(\pi_{\alpha}^{1}, \pi_{\alpha}^{2}\right), \quad\left(\bar{\pi}_{\dot{\alpha}}^{+}, \bar{\pi}_{\dot{\alpha}}^{-}\right)=\left(\bar{\pi}_{\dot{\alpha} 2},-\bar{\pi}_{\dot{\alpha} 1}\right),
$$

and by redefining the component fields, we can rewrite the expression $(3.77)$ in $\operatorname{SL}(2, \mathbb{C})$ covariant form, but we would like to stress that the spin content in the expansion (3.77) is degenerate. This degeneracy can be however removed by the harmonic condition on the wave function (see also [71])

$$
D^{++} \tilde{\Psi}^{(+)}=0
$$

Since that the monomials $v_{\left(i_{1}\right.}^{+} \ldots v_{i_{N}}^{+} v_{j_{1}}^{-} \ldots v_{\left.j_{K}\right)}^{-}$form the basis, as a solution of (3.81), we obtain the following wave function

$$
\tilde{\Psi}^{(+)}\left(h_{\alpha}{ }^{i}, v_{\dot{i}}^{ \pm}\right)=\sum_{N=0}^{\infty} v_{\dot{i}_{1}}^{+} \ldots v_{\dot{i}_{N}}^{+} f^{\dot{i}_{1} \ldots \dot{i}_{N}}(h) .
$$

This twistor wave function rewritten in Lorentz covariant way takes the form

$$
\tilde{\Psi}^{(+)}\left(\pi_{\alpha}^{ \pm}, \bar{\pi}_{\dot{\alpha}}^{ \pm}\right)=\sum_{N=0}^{\infty} \pi_{\alpha_{1}}^{+} \ldots \pi_{\alpha_{N}}^{+} \psi^{\alpha_{1} \ldots \alpha_{N}}\left(p_{\mu}\right) .
$$

Note that the twistor wave function (3.83) also depends on $\pi_{\alpha}^{-}$and $\bar{\pi}_{\dot{\alpha}}^{ \pm}$through $p_{\mu}$ in the argument of the component fields.

Spin $s=L / 2$ massive particles are described by the fields $\psi^{\alpha_{1} \ldots \alpha_{L}}\left(p_{\mu}\right)$. The corresponding spacetime fields are obtained by an integral Fourier-twistor transform which combines the Fourier and twistor transformations. More explicitly, by means of these integral transformations we can obtain the following multispinor fields, all with a total of $L$ undotted plus dotted indices,

$$
\begin{aligned}
\phi_{\alpha_{1} \ldots \alpha_{L}}(x)= & \int d^{6} \pi e^{-i x^{\mu} p_{\mu}} \pi_{\alpha_{1}}^{-} \ldots \pi_{\alpha_{L}}^{-} \tilde{\Psi}^{(+)}\left(\pi^{ \pm}, \bar{\pi}^{ \pm}\right) \\
\phi_{\alpha_{1} \ldots \alpha_{L-1}} \dot{\beta}_{1} & =\int d^{6} \pi e^{-i x^{\mu} p_{\mu}} \pi_{\alpha_{1}}^{-} \ldots \pi_{\alpha_{L-1}}^{-} \bar{\pi}^{-\dot{\beta}_{1}} \tilde{\Psi}^{(+)}\left(\pi^{ \pm}, \bar{\pi}^{ \pm}\right) \\
\phi_{\alpha_{1} \ldots \alpha_{L-2}} \dot{\beta}_{1} \dot{\beta}_{2} & =\int d^{6} \pi e^{-i x^{\mu} p_{\mu}} \pi_{\alpha_{1}}^{-} \ldots \pi_{\alpha_{L-2}}^{-} \bar{\pi}^{-\dot{\beta}_{1}} \bar{\pi}^{-\dot{\beta}_{2}} \tilde{\Psi}^{(+)}\left(\pi^{ \pm}, \bar{\pi}^{ \pm}\right), \\
\ldots & \\
\phi^{\dot{\beta}_{1} \ldots \dot{\beta}_{L}}(x)= & \int d^{6} \pi e^{-i x^{\mu} p_{\mu}} \bar{\pi}^{-\dot{\beta}_{1}} \ldots \bar{\pi}^{-\dot{\beta}_{L}} \tilde{\Psi}^{(+)}\left(\pi^{ \pm}, \bar{\pi}^{ \pm}\right)
\end{aligned}
$$


where $p_{\mu}$ is defined by (3.4) as a bilinear product of twistors. In the integrals (3.84) for a given $L$, only the term $\pi_{\alpha_{1}}^{+} \ldots \pi_{\alpha_{L}}^{+} \psi^{\alpha_{1} \ldots \alpha_{L}}\left(p_{\mu}\right)$ in the twistorial wave function (3.83), with $\mathrm{U}(1)$ harmonic charge $q=L$ (see [71]), gives a non-zero contribution. Note that $d^{6} \pi=d^{3} h d^{3} v$ where $d^{3} v$ is the harmonic measure on $\mathrm{SU}(2)$ manifold whereas the measure on the Lobachevski mass hyperboloid $d^{3} h=d \Omega$ may be written as $d \Omega=d^{3} \vec{p} /\left(2 p_{0}\right)$ after using the the relation (3.72). Since all fields in eqs. (3.84) are derived from eq. (3.83), they have to be related. Denoting by $N(M)$ the total number of undotted (dotted) indices, $L=N+M$, we can show that the multispinors $\phi_{\alpha_{1} \ldots \alpha_{N}} \dot{\beta}_{1} \ldots \dot{\beta}_{M}\left(=\phi_{(M, N)}\right.$ for short) in (3.84), symmetric in both the $\alpha$ and the $\dot{\beta}$ indices, satisfy the following sequence of Dirac-FierzPauli field equations

$$
\begin{aligned}
i \partial_{\alpha \dot{\beta}_{M}} \phi_{\alpha_{1} \ldots \alpha_{N}} \dot{\beta}_{1} \ldots \dot{\beta}_{M} & =m \phi_{\alpha \alpha_{1} \ldots \alpha_{N}} \dot{\beta}_{1} \ldots \dot{\beta}_{M-1} \\
i \partial^{\dot{\beta}_{M} \alpha} \phi_{\alpha \alpha_{1} \ldots \alpha_{N}} \dot{\beta}_{1} \ldots \dot{\beta}_{M-1} & =m \phi_{\alpha_{1} \ldots \alpha_{N}} \dot{\beta}_{1} \ldots \dot{\beta}_{M}
\end{aligned}
$$

where $\partial_{\alpha \dot{\beta}}=\left(\sigma^{\mu}\right)_{\alpha \dot{\beta}} \partial_{\mu}, \partial^{\dot{\beta} \alpha}=\left(\tilde{\sigma}^{\mu}\right)^{\dot{\beta} \alpha} \partial_{\mu}$ and $\partial_{\beta \dot{\gamma}} \partial^{\dot{\gamma} \alpha}=\delta_{\beta}^{\alpha} \square, \partial^{\dot{\alpha} \gamma} \partial_{\gamma \dot{\beta}}=\delta_{\dot{\beta}}^{\dot{\alpha}} \square$. Notice that, for a given $L$, the multispinor field $\phi_{(N, M)}$ contains the sequence of spins $\left(\frac{N+M}{2}=\frac{L}{2}, \ldots, \frac{|N-M|}{2}\right)$, as it follows by looking at the $\mathrm{SU}(2)$ representation contents of the finite irreducible representations of $\mathrm{SL}(2, \mathbb{C})$ (see e.g. [73]). The nonmaximal $\left(s<\frac{L}{2}\right)$ spins are eliminated subjecting $\phi_{(N, M)}$ to the generalized Lorenz conditions

$$
\partial^{\dot{\beta} \alpha} \phi_{\alpha_{1} \ldots \alpha_{N-1} \alpha \dot{\beta}} \dot{\beta}_{1 \ldots} \dot{\beta}_{M-1}=0
$$

which follow as well from the formulae (3.84), plus all the tracelessness conditions which are also consequences of (3.84).

Dirac-Fierz-Pauli equations for spin $s$ can be written in Weyl spinor notation as equations relating the $\phi_{(L, 0)}$ and $\phi_{(L-1,1)}$ multispinor fields

$$
\begin{aligned}
i \partial_{\alpha \dot{\beta}} \phi_{\alpha \alpha_{1} \ldots \alpha_{L-1}} \dot{\beta} & =m \phi_{\alpha \alpha_{1} \ldots \alpha_{L-1}}, \\
i \partial^{\dot{\beta} \alpha} \phi_{\alpha \alpha_{1} \ldots \alpha_{L-1}} & =m \phi_{\alpha_{1} \ldots \alpha_{L-1}} \dot{\beta} ;
\end{aligned}
$$

alternatively, we can choose eq. (3.85) for the multispinors $\phi_{(0, L)}$ and $\phi_{(1 . L-1)}$. The second equation in (3.87) can be considered (for $m \neq 0$ ) as defining the fields $\phi_{(L-1,1)}$, so the whole set of fields (3.84) can be obtained from the fields $\phi_{(L, 0)}$, which satisfy the massive Klein-Gordon equation and describe spins $s=L / 2[73,74]$. Indeed, using relations (3.85) subsequently for the fields $\phi_{(L-1,1)}, \ldots, \phi_{(L-M, M)}$ it may be shown that all these fields can be expressed in terms of $\phi_{(L, 0)}$ by

$$
\begin{aligned}
\phi_{\alpha_{1} \ldots \alpha_{L-M}} \dot{\beta}_{1} \ldots \dot{\beta}_{M} & =\frac{i}{m} \partial^{\dot{\beta}_{M} \alpha_{L-M+1}} \phi_{\alpha_{1} \ldots \alpha_{L-M+1}} \dot{\beta}_{1} \ldots \dot{\beta}_{M-1}=\ldots= \\
& =\left(\frac{i}{m}\right)^{M} \partial^{\left(\dot{\beta}_{M} \alpha_{L-M+1}\right.} \partial^{\dot{\beta}_{M-1} \alpha_{L-M+2}} \ldots \partial^{\left.\dot{\beta}_{1}\right) \alpha_{L}} \phi_{\alpha_{1} \ldots \alpha_{L}} .
\end{aligned}
$$

It follows therefore that all the field equations for $\phi_{(N, M)}(N+M=L)$ in (3.85) can be obtained from an independent pair of linear HS field equations for $\phi_{(N, M)}=\phi_{(L-1,1)}$ and $\phi_{(N, M)}=\phi_{(0, L)}$ or from $\phi_{(L, 0)}$ and $\phi_{(1, L-1)}$. In particular if we choose $L=1$ in (3.87), we 
obtain the standard Dirac equation for a Dirac field in the Weyl realization as the sum of an undotted spinor and a dotted one, $\phi_{(1,0)} \oplus \phi_{(0,1)}$ in the our notation.

If $L=2$ we obtain the Proca equations expressed in terms of $\phi_{(1,1)}, \phi_{(2,0)}$ and $\phi_{(0,2)}$. Consider first (3.85) for $N=0, M=2$,

$$
i \partial_{\alpha_{1} \dot{\beta}_{1}} \phi^{\dot{\beta}_{1} \dot{\beta}_{2}}=m \phi_{\alpha_{1}} \dot{\beta}_{2}, \quad i \partial^{\dot{\beta}_{1} \alpha} \phi_{\alpha}^{\dot{\beta}_{2}}=m \phi^{\dot{\beta}_{1} \dot{\beta}_{2}} .
$$

Eliminating $\phi^{\dot{\beta}_{1} \dot{\beta}_{2}}$, we see that the vector field $\phi_{\alpha_{1} \dot{\beta}_{2}}$ satisfies the massive Klein-Gordon equation. Further, using the symmetry in the $\beta_{1} \beta_{2}$ indices, it follows that the two equations above imply the Lorenz condition (see (3.86))

$$
\partial^{\dot{\beta} \alpha} \phi_{\alpha \dot{\beta}}=0
$$

which eliminates the spin zero part of $\phi_{\alpha \dot{\beta}}$. Thus, by virtue of eqs. (3.89), $\phi_{\alpha \dot{\beta}}$ is the spin one Proca field $\phi_{\mu}$ satisfying $\left(\square+m^{2}\right) \phi^{\mu}=0, \partial_{\mu} \phi^{\mu}=0$ (see eq. (A.15)). Similarly, if we now consider the case $N=1=M$ in eqs. (3.85), we obtain

$$
i \partial_{\alpha_{1} \dot{\beta}} \phi_{\alpha_{2}}{ }^{\dot{\beta}}=m \phi_{\alpha_{1} \alpha_{2}}, \quad i \partial^{\dot{\beta} \alpha_{1}} \phi_{\alpha_{1} \alpha_{2}}=m \phi_{\alpha_{2}}{ }^{\dot{\beta}} .
$$

As before, the Klein-Gordon equation and the Lorenz condition for the four-vector field $\phi_{\alpha \dot{\beta}}$ are contained in eqs. (3.91), which again reproduce the equations satisfied by a Proca field.

We note that to obtain the Proca equations as a massive extension of the Maxwell equations it is sufficient to describe the free field dynamics in terms of the field strength $\phi_{\mu \nu}=\partial_{\mu} \phi_{\nu}-\partial_{\nu} \phi_{\mu}$. The tensor $\phi_{\mu \nu}$ may be expressed in terms of its dual and antiselfdual parts, $\phi_{\mu \nu} \sim\left(\phi_{(2,0)}, \phi_{(0,2)}\right)$. Using these two bispinor fields one obtains the Proca equations, $\partial^{\mu} \phi_{\mu \nu}+m^{2} \phi_{\nu}=0$.

\section{Outlook}

We have presented in this paper new massive particle models in $D=3$ and $D=4$ spacetimes enlarged in $D=3$ by two $\left(y_{r}^{\alpha \beta}, r=1,2\right)$ or in $D=4$ by three $\left(y_{r}^{\alpha \dot{\beta}}, r=\right.$ $1,2,3)$ additional copies of Minkowskian four-vector variables and their momenta. After quantization, the wave functions are defined on $\mathrm{SL}(2 ; \mathbb{K})$ manifolds $(\mathbb{K}=\mathbb{R}$ for $D=3$, $\mathbb{K}=\mathbb{C}$ for $D=4$ ) and describe towers of free massive HS fields. A natural extension of these models is the $D=6$ case, in which the wave functions would be defined on the $\mathrm{SL}(2 ; \mathbb{H})$ manifold, with 12 real parameters. In such a case, the complex $D=4$ twistors in section 3 should be replaced by quaternionic $D=6$ twistors (see e.g. [75]), defined as fundamental spinorial realization of the $D=6$ conformal $\mathrm{SO}(6,2)$ group with spinorial quaternionic covering $U_{\alpha}(4 ; \mathbb{H}) \simeq O^{*}(8 ; \mathbb{C})$ group (see e.g. $[15,76]$ ).

We would like to point out that it is possible to relate the $D=3$ and $D=4$ massive models with $D=4$ and $D=5$ massless ones by observing that massless fields in $D+$ 1 spacetimes become massive in one less dimension $D$ after dimensional reduction and interpreting the $(D+1)$-th momentum component as the mass in $D$-dimensional spacetime. There is a link between the description of helicity in massless theories and spin in massive case; i.e.

$$
(D+1) \text { 'helicity' } \longrightarrow \quad D \text { 'spin' }
$$


In particular, the Abelian helicity operator in $D=4$ (see (1.8)) corresponds to the spin operator (2.11) in $D=3$ and further the $\mathrm{SU}(2)$ spin algebra in $D=4$ could be used analogously to describe the generalized helicity states in $D=5$. We add that recently it has been pointed out that the symplectic two-form describing the spin contribution to the $D=3$ free massive spinning particle dynamics [43] can be identified with the symplectic two-form describing the helicity part of particle dynamics for massless $D=4$ particles with nonvanishing helicity [77].

One can extend our considerations to the supersymmetric case. In it, as in the first example of a spinorial particle model in tensorial spacetime extended to superspace [3], the additional variables can be associated with the so-called tensorial central charges of the supersymmetry algebras (i.e., central but for the Lorentz subalgebra). These charges play an important role in the theory of supersymmetric extended objects [78]; the corresponding central tensorial generators of the superlagebras act as differential operators on the additional coordinates of the associated extended superspaces. ${ }^{10}$ From this perspective, the two-twistor models introduced here can be related with the tensorial central charges of $N=2$ supersymmetry and the variables of the suitably extended superspaces. The most general $D=3 N=2$ superalgebra extended by tensorial central charges is as follows

$$
\left\{Q_{\alpha}^{i}, Q_{\beta}^{j}\right\}=\delta^{i j} P_{\alpha \beta}+\left(\sigma_{1}\right)^{i j} Z_{\alpha \beta}^{(1)}+\left(\sigma_{3}\right)^{i j} Z_{\alpha \beta}^{(2)}+\epsilon^{i j} \epsilon_{\alpha \beta} \tilde{Z} .
$$

The real vectorial 'central' charges $Z_{\alpha \beta}^{(1)}, Z_{\alpha \beta}^{(2)}$ may be considered as the momenta $p_{\alpha \beta}^{r}$ generating the translations of our additional coordinates $y_{r}^{\alpha \beta}(r=1,2$; see (2.12) and (2.19)). In this view, the first formula (2.21) takes the form

$$
Z_{\alpha \beta}^{(r)}=-i \frac{\partial}{\partial y_{r}^{\alpha \beta}} .
$$

In the $D=4, N=2$ supersymmetry algebra with tensorial central charges, the generators associated with the coordinates listed in (1.9) (see also section 3) appear as part of those of the extended superalgebra

$$
\begin{aligned}
& \left\{Q_{\alpha}^{i}, \bar{Q}_{\dot{\beta}}^{j}\right\}=\left(\sigma_{a}\right)^{i j} P_{\alpha \dot{\beta}}{ }^{a}, \\
& \left\{Q_{\alpha}^{i}, Q_{\beta}^{j}\right\}=\delta^{i j} \tilde{Z}_{\alpha \beta}+\left(\sigma_{1}\right)^{i j} \tilde{Z}_{\alpha \beta}^{(1)}+\left(\sigma_{3}\right)^{i j} \tilde{Z}_{\alpha \beta}^{(2)}+\epsilon^{i j} \epsilon_{\alpha \beta} \tilde{Z}
\end{aligned}
$$

(similarly for $\left(\left\{\bar{Q}_{\dot{\alpha}}^{i}, \bar{Q}_{\dot{\beta}}^{j}\right\}\right)$, where the 16 generators $P_{\alpha \dot{\beta}}^{a}$ are real and the 10 generators $\tilde{Z}_{\alpha \beta}$, $\tilde{Z}_{\alpha \beta}^{(1)}, \tilde{Z}_{\alpha \beta}^{(2)}, \tilde{Z}$ are complex (i.e. there are 36 bosonic real generators). In our $D=4$ model we have only used the sixteen coordinates $y_{\alpha \dot{\beta}}^{a}$ (see eq. (2.12)) associated with $P_{\alpha \dot{\beta}}^{a}, a=0,1,2,3$ and the remaining 10 complex tensorial charges were put equal to zero. Let us observe that for $N=1$ only the first term in the r.h.s. of the relation (4.5) survives and describes the tensorial central charges used in $[2-5]$. If $N=2$ we also note that the generator $\tilde{Z}$ in (4.2) and (4.5) that we did not include in our considerations is a truly central one (it is a Lorentz scalar). This generator, associated with a scalar central coordinate, has

\footnotetext{
${ }^{10}$ For a discussion of the role of additional coordinates of extended superspaces see $[79,80]$ and references therein.
} 
eigenvalues characterizing the mass; its role in $N=2$ massive superparticle model was elucidated long ago [81, 82].

The models discussed in this paper give the same mass for all HS fields, which of course is very restrictive. In a physical HS case, when considering e.g. spin excitations in string theory, the masses are spin-dependent. They lie on a Regge trajectory, which in the general case can be described by replacing the constant mass by an spin-dependent function $m=m(s)$ (usually linear). In this case, the constant $m$ in the mass-shell condition should be replaced by a spin-dependent operator (see (3.13), (3.14), (3.50)) i.e.,

$$
m^{2} \quad \rightarrow \quad m^{2}\left(\vec{S}^{2}\right) .
$$

In the twistor formulation, the spinorial mass shell conditions (eq. (1.10) in $D=4$ ) may be considered as 'complex roots' of the standard mass shell condition. It is an interesting problem to see how to introduce, in the complex mass parameter $M$ appearing in eqs. (1.10), a dependence on the twistor variables that could lead to HS multiplets with masses on a Regge trajectory.

Another problem worth studying is the description of interacting massive HS theories. To this aim, one could follow the Fradkin-Vasiliev prescription for massless HS fields [83] and introduce in our formalism the AdS radius i.e., one could generalize the set of coordinates $\left(x_{\mu}, y_{\mu}^{r}\right)$ (see (1.9)) to the case where $x_{\mu}$ is endowed with a constant spacetime curvature. However, in the interacting massive HS theory, the finite AdS radius is not necessary because it is possible to rescale the derivatives in higher order terms by using the mass parameter. Indeed, the cosmological constant $\Lambda$ and mass parameter $m$ play an analogous role in the field equations, as reflected e.g. in the shift $m^{2} \rightarrow m^{2} \pm \frac{3}{2} \Lambda^{2}$ (see e.g. [37]) which appears when the KG equation is formulated in $(A) d S$ spacetime. ${ }^{11}$ Theefore, one can conclude that massive interacting HS theories should already exist on a flat Minkowski background.

The interacting massless HS theory is usually described as a HS theory, also called HS gravity, ${ }^{12}$ with nonlinearities generated e.g. by non-Abelian HS field strengths. In such framework the mass can be introduced in two ways.

1) In the first one, the mass parameter appears as a consequence of the spontaneous symmetry breaking of HS local gauge symmetries, due to the coupling of the HS fields to supplementary Stueckelberg fields carrying spontaneously broken local gauge degrees of freedom [89-94]. In the Vasiliev formalism (see e.g. [26, 30, 31]), the HS gauge connection fields are given by a vectorial master field $\omega_{\mu}\left(x ; y_{i}^{\alpha}, \bar{y}^{\dot{\alpha} i}\right)$; a scalar master field $C\left(x ; y_{i}^{\alpha}, \bar{y}^{\dot{\alpha} i}\right)$ encodes, besides the gauge-invariant HS curvatures, new degrees of freedom that describe the low $\operatorname{spin}\left(s=0\right.$ and $\left.s=\frac{1}{2}\right)$ matter. The introduction of a Stueckelberg-Higgs mechanism requires new local symmetries described by a new pure gauge scalar master field

\footnotetext{
${ }^{11}$ The problem with the limit $m \rightarrow 0$ for flat $(\Lambda=0)$ HS fields is also reflected in the appearance of Van Dam-Veltman-Zakharov discontinuity [84, 85] in massive gravity models. However, if $\Lambda \neq 0$, in the limit $m \rightarrow 0$ such a discontinuity does not exist [86, 87].

${ }^{12}$ We recall that gravity can be described as gauge theory of massless $s=2$ fields, with local gauge transformations realized as spacetime diffeomorphism (see e.g. [88]).
} 
$\widetilde{C}\left(x ; y_{i}^{\alpha}, \bar{y}^{\dot{\alpha} i}\right)$. This field accounts for the set of spontaneously broken local gauges given as covariantized shifts of the spacetime fields defined by the spinorial Taylor expansion of $\widetilde{C}\left(x ; y_{i}^{\alpha}, \bar{y}^{\dot{\alpha} i}\right)$. The masses for the the various spins are obtained through the particular gauge fixings that replace the spacetime dependent field components of $\widetilde{C}\left(x ; y_{i}^{\alpha}, \bar{y}^{\dot{\alpha} i}\right)$ by parameters.

The idea of obtaining nonvanishing mass via Stueckelberg-Higgs mechanism in massless HS gauge theory was also proposed in (super)string theory to generate an infinite collection of massive states which lie on Regge trajectories. Recently a generalization of HS algebras has been proposed under the name of multiparticle extension of HS symmetries $[95,96]{ }^{13}$ This permits to look at a string as an infinite collection of interacting HS multiplets which, in a miraculous way, seem to provide a finite (or at least renormalizable) example of interacting massive HS field theory.

2) In the second case, the mass parameter in the massive HS theory produces a "hard" breaking of local HS gauge symmetries and the massive HS fields may be considered as describing the sector of non-gauge HS matter, entering in HS gravity equations at the r.h.s. of HS-extended Einstein equations. These HS fields would be related with HS massive currents, which for massless HS fields are described by current master fields of rank two with double number of spinorial coordinates [23, 25, 26, 96]. This last property is also a feature of our massive master HS fields, with double number of twistorial coordinates. This suggests that the massive currents could be introduced in a natural way by using a master field analogue of the old current-field identities idea [100, 101].

The current master fields describe the "conformal side" in the realization of HS/CFT duality in Vasiliev theory [23-26, 96]. As shown by Flato and Fronsdal [102], the binary products of $D=3$ singletons describe the $D=4 \rightarrow D=3$ reduction of the $D=4$ multiplet of free AdS HS fields; analogously, in HS field theory the bilinear products of $D=3$ massless master fields describe the conserved HS currents that can be identified with the $D=3$ holomorphic boundary of the $D=4$ massless AdS master field. For massive HS fields the conformal HS symmetry is broken, which leads to the nonconservation of the HS conformal currents and the deformation of HS AdS/CFT duality picture that is obtained for massless HS theory. The way in which the nonvanishing mass of HS fields modifies the known massless HS AdS/CFT duality scheme will be a subject for our future research.

In sections 2 and 3 we considered wave functions depending on the spinors $\lambda_{\alpha}^{i}$ in $D=3$ and $\pi_{\alpha}^{i}$ in $D=4$. These spinorial momentum coordinates, due to the constraints (2.6) and (1.10), describe $D=3$ and $D=4$ Lorentz group manifolds. Considering the differential realization of the spinorial variables, $\hat{\lambda}_{\alpha}^{i}=-i \partial / \partial y_{i}^{\alpha}$ in $D=3$ and $\hat{\pi}_{\alpha}^{i}=-i \partial / \partial y_{i}^{\alpha}$ in $D=4$, we can compare our results with the unfolding equations for free massless fields [3-5]. In the massless case, following [1], one considers a tensorial extension of spacetime $x^{\mu} \rightarrow\left(x^{\mu}, y^{[\mu \nu]}\right)$ that permits the introduction of $\operatorname{Sp}(8)$-covariant fields in Minkowski space with all $D=4$

\footnotetext{
${ }^{13}$ The standard HS algebras are described by the linear basis of various enveloping Heisenberg algebras, with canonical generators represented by quantized vectorial or spinorial (twistorial) coordinates (see e.g. [97-99]). The multiparticle extension of HS algebra is described as "doubly infinite" enveloping standard HS algebras.
} 
helicities belonging to an irreducible $\operatorname{Sp}(8)$ representation. In our massive model, the extension $x^{\mu} \rightarrow\left(x^{\mu}, y_{r}^{\mu}\right)$ of spacetime is linked to the introduction of Lorentz-covariant $\mathrm{SU}(2)$ frames (see e.g. (3.7), (3.8)) describing all $D=4$ spin degrees of freedom. ${ }^{14}$ However, both extensions of the $D=4$ Minkowski spacetime described above are rather introduced by symmetry arguments and, in both cases, these extensions are not mandatory to define the free dynamics of HS master fields. If we eliminate the auxiliary spacetime variables by solving the auxiliary unfolding equations ((3.27) with $a=1,2,3$ in massive case), we are left with a truncated form of the unfolding equation with only standard spacetime derivatives. In the massive case the free HS master fields are described by the unfolded equation (3.27) for $a=0$ ( $y_{0} \dot{\beta} \alpha$ are the spacetime coordinates) supplemented with the mass quantum constraints (1.10):

$$
\begin{aligned}
\left(i \partial_{\alpha \dot{\beta}}-\frac{\partial^{2}}{\partial y_{i}^{\alpha} \partial \bar{y}^{\dot{\beta} i}}\right) \Psi(x, y, \bar{y}) & =0, \\
\left(\frac{\partial^{2}}{\partial y_{i}^{\alpha} \partial y_{\alpha}^{i}}-2 M\right) \Psi(x, y, \bar{y}) & =0, \quad\left(\frac{\partial^{2}}{\partial \bar{y}_{i}^{\dot{\alpha}} \partial \bar{y}_{\dot{\alpha}}^{i}}-2 \bar{M}\right) \Psi(x, y, \bar{y})=0 .
\end{aligned}
$$

Equations (4.7) and (4.8) describe free massive HS fields in flat Minkowski space; by a suitable (A)dS covariantization of the vectorial and spinorial derivatives in (4.7), (4.8) the master field equations describing free massive HS fields in (A)dS spacetime may be obtained. Further, one can study the couplings of massive HS fields with full HS gravity background as well as dynamical gauge fields.

Finally, we note that the use of a generalized spacetime with vector variables going beyond the standard spacetime vector is also an important ingredient of the BRST approach to the Lagrangian formulation of HS fields ${ }^{15}$ developed in [105-108].

\section{Acknowledgments}

The authors would like to thank M.A. Vasiliev for valuable remarks. This paper has been partially supported by research grants from the Spanish MINECO (CONSOLIDER CPAN-CSD2007-00042) as well as by the Polish National Science Center projects 2013/09/B/ST2/02205, 2014/13/B/ST2/04043 and Maestro grant 2013/10/A/ST2/00106. S.F. acknowledges support from the RFBR grants 12-02-00517, 13-02-90430 and a grant of the Bogoliubov-Infeld Programme.

\section{A Notation}

$\boldsymbol{D}=\mathbf{3}$ spacetime. The spacetime metric is $\eta_{\mu \nu}=\operatorname{diag}(+1,-1,-1)$. Dirac spinor indices are labeled by $\alpha=1,2$ and we use mostly $D=3$ real Majorana spinors. In particular, the twistor variables $\left(\lambda_{\alpha}^{i}\right)^{*}=\lambda_{\alpha}^{i}, i=1,2$ are real.

\footnotetext{
${ }^{14}$ The introduction of Lorentz frames to describe spin kinematics goes back to Souriau [103, 104]. Unfortunately we were not able to introduce the manifold $\left(x^{\mu}, y_{r}^{\mu}\right)$ in a group-theoretical way, as e.g. $\left(x^{\mu}, y^{[\mu \nu]}\right)$ can be defined as a parabolic coset of $\operatorname{Sp}(8)[4,5]$.

${ }^{15} \mathrm{SF}$ thanks I.L. Buchbinder for a clarifying discussion about this approach. BRST techniques are not considered in this paper.
} 
We use the following real Majorana realization for the $\gamma$-matrices:

$$
\begin{aligned}
\left\{\gamma^{\mu}, \gamma^{\nu}\right\} & =-2 \eta^{\mu \nu}, \\
\left(\gamma_{\mu}\right)_{\alpha}{ }^{\beta}: \quad \gamma_{0} & =i \sigma_{2}, \quad \gamma_{1}=\sigma_{1}, \quad \gamma_{2}=\sigma_{3},
\end{aligned}
$$

where $\sigma_{1}, \sigma_{2}, \sigma_{3}$ are the usual Pauli matrices. In this realization the antisymmetric the charge conjugation matrix $C_{\alpha \beta}=\epsilon_{\alpha \beta}$ coincides with the matrix $\gamma^{0}$. Thus, spinor indices are raised and lowered by $\lambda^{\alpha i}=\epsilon^{\alpha \beta} \lambda_{\beta}^{i}, \lambda_{\alpha}^{i}=\epsilon_{\alpha \beta} \lambda^{\beta i}$, where $\epsilon_{12}=\epsilon^{21}=1$. The matrices

$$
\left(\gamma_{\mu}\right)_{\alpha \beta}=\epsilon_{\beta \gamma}\left(\gamma_{\mu}\right)_{\alpha}^{\gamma}: \quad\left(\gamma_{0}\right)_{\alpha \beta}=\mathbf{1}_{2}, \quad\left(\gamma_{1}\right)_{\alpha \beta}=\sigma_{3}, \quad\left(\gamma_{2}\right)_{\alpha \beta}=\sigma_{1}
$$

form a basis for the $2 \times 2$ symmetric matrices. In particular,

$$
\left(\gamma^{\mu}\right)_{\alpha \beta}\left(\gamma_{\nu}\right)^{\alpha \beta}=2 \delta_{\nu}^{\mu}
$$

As a result $\left(A_{\mu}\right.$ and $B_{\mu}$ are three-vectors)

$$
A_{(\alpha \beta)}=\frac{1}{\sqrt{2}} A_{\mu}\left(\gamma^{\mu}\right)_{\alpha \beta}, \quad A_{\mu}=\frac{1}{\sqrt{2}} A_{\alpha \beta}\left(\gamma_{\mu}\right)^{\alpha \beta},
$$

and

$$
A^{\mu} B_{\mu}=A^{\alpha \beta} B_{\alpha \beta}
$$

In section 2.3 we also use a complex representation of $D=3$ Dirac-Clifford algebra, which is obtained from the Majorana realization (A.2) by the similarity transformation (2.37): $\gamma_{\mu} \rightarrow U \gamma_{\mu} U^{-1}$. In such a realization of $D=3$ Dirac algebra we use $\operatorname{SU}(1,1)$ as the $\operatorname{Spin}(2,1)$ group, and the $\gamma$-matrices take the form

$$
\begin{aligned}
& \left(\gamma_{\mu}\right)_{\alpha}{ }^{\beta}: \quad \gamma_{0}=i \sigma_{3}, \quad \gamma_{1}=\sigma_{1}, \quad \gamma_{2}=-\sigma_{2}, \\
& \left(\gamma_{\mu}\right)_{\alpha \beta}=\epsilon_{\beta \gamma}\left(\gamma_{\mu}\right)_{\alpha}^{\gamma}: \quad\left(\gamma_{0}\right)_{\alpha \beta}=-i \sigma_{1}, \quad\left(\gamma_{1}\right)_{\alpha \beta}=\sigma_{3}, \quad\left(\gamma_{2}\right)_{\alpha \beta}=i \mathbf{1}_{2} \text {. }
\end{aligned}
$$

We will proceed similarly for matrices with internal $i, j$ indices. In particular, $\lambda_{\alpha}^{i}=$ $\epsilon^{i j} \lambda_{\alpha j}, \lambda_{\alpha i}=\epsilon_{i j} \lambda_{\alpha}^{j}$, where $\epsilon_{i j}$ and $\epsilon^{i j}$ are defined by $\epsilon_{12}=\epsilon^{21}=1$. Also, we will use the matrices $(a=0,1,2)$ acting of internal indices

$$
\begin{array}{rlll}
\left(\gamma_{a}\right)_{i}{ }^{j}: & \gamma_{0}=i \sigma_{2}, & \gamma_{1}=\sigma_{1}, & \gamma_{2}=\sigma_{3} \\
\left(\gamma_{a}\right)_{i j}=\epsilon_{j k}\left(\gamma_{a}\right)_{i}{ }^{k}: & \gamma_{0}=\mathbf{1}_{2}, & \gamma_{1}=\sigma_{3}, & \gamma_{2}=-\sigma_{1}
\end{array}
$$

$\boldsymbol{D}=4$ spacetime. The spacetime metric is $\eta_{\mu \nu}=\operatorname{diag}(+1,-1,-1,-1)$. We shall use the two-component Weyl spinor notation. In particular, four-vector quantities are defined in terms of spinors as $x_{\alpha \dot{\beta}}=x_{\mu} \sigma_{\alpha \dot{\beta}}^{\mu}$, where

$$
\left(\sigma_{\mu}\right)_{\alpha \dot{\beta}}=\left(\mathbf{1}_{\mathbf{2}} ; \sigma_{1}, \sigma_{2}, \sigma_{3}\right)_{\alpha \dot{\beta}}
$$

and $\sigma_{1}, \sigma_{2}, \sigma_{3}$ are the Pauli matrices. Spinor indices are raised and lowered by $\epsilon_{\alpha \beta}, \epsilon^{\alpha \beta}$, $\epsilon_{\dot{\alpha} \dot{\beta}}, \epsilon^{\dot{\alpha} \dot{\beta}}$ with nonvanishing components $\epsilon_{12}=-\epsilon_{21}=\epsilon^{21}=-\epsilon^{12}=1$. As the result, the matrices

$$
\left(\tilde{\sigma}_{\mu}\right)^{\dot{\alpha} \beta}=\epsilon^{\dot{\alpha} \dot{\delta}} \epsilon^{\beta \gamma}\left(\sigma_{\mu}\right)_{\gamma \dot{\delta}}=\left(\mathbf{1}_{2} ;-\sigma_{1},-\sigma_{2},-\sigma_{3}\right)^{\dot{\alpha} \beta}
$$


satisfy

$$
\sigma_{\alpha \dot{\gamma}}^{\mu} \tilde{\sigma}^{\nu \dot{\gamma} \beta}+\sigma_{\alpha \dot{\gamma}}^{\mu} \tilde{\sigma}^{\nu \dot{\gamma} \beta}=2 \eta^{\mu \nu} \delta_{\alpha}^{\beta}, \quad \sigma_{\alpha \dot{\beta}}^{\mu} \tilde{\sigma}_{\nu}^{\dot{\beta} \alpha}=2 \delta_{\nu}^{\mu} .
$$

The Dirac matrices are given by

$$
\gamma_{\mu}=\left(\begin{array}{cc}
0 & \sigma_{\mu} \\
-\tilde{\sigma}_{\mu} & 0
\end{array}\right), \quad\left\{\gamma^{\mu}, \gamma^{\nu}\right\}=-2 \eta^{\mu \nu} .
$$

The link between Minkowski four-vectors and spinorial quantities is given by

$$
A_{\alpha \dot{\beta}}=\frac{1}{\sqrt{2}} A_{\mu}\left(\sigma^{\mu}\right)_{\alpha \dot{\beta}}, \quad A_{\mu}=\frac{1}{\sqrt{2}} A_{\alpha \dot{\beta}}\left(\tilde{\sigma}_{\mu}\right)^{\dot{\beta} \alpha},
$$

so that

$$
A^{\mu} B_{\mu}=A^{\alpha \dot{\beta}} B_{\alpha \dot{\beta}}
$$

Similar matrices are used in internal space with indices $i, j$. At this point it is necessary to make a comment. There are two methods to indicate the complex conjugate spinor representation. The first one uses dotted indices as in (A.11). The second method, often used for $\mathrm{SU}(2)$, raises and lowers two-spinor indices. We use the second method for matrices in internal space. So, we use matrices

$$
\left(\sigma_{a}\right)_{i}{ }^{j}=\left(\sigma_{0} ; \sigma_{r}\right)_{i}{ }^{j}=\left(\mathbf{1}_{\mathbf{2}} ; \sigma_{1}, \sigma_{2}, \sigma_{3}\right)_{i}{ }^{j} .
$$

In these matrices indices are raised and lowered by $\epsilon_{i j}$ and $\epsilon^{i j}$ with components $\epsilon_{12}=\epsilon^{21}=$ 1 ; under complex conjugation the position of these indices is exchanged e.g., $\left(\pi_{\alpha}^{i}\right)^{*}=\bar{\pi}_{\dot{\alpha} i}$.

\section{B From $D=3$ spinorial to $D=4$ vectorial particle model}

The action (2.12) proposed in this paper in the case $c=1$ becomes $\overline{\mathrm{SO}(2,2)}=\overline{\mathrm{SO}(2,1)} \otimes$ $\overline{\mathrm{SO}(2,1)_{\text {int }}}=\mathrm{SL}(2 ; \mathbb{R}) \otimes \mathrm{SL}(2 ; \mathbb{R})_{\text {int }}$-invariant, where the indices $\alpha, \beta$ describe the $D=3$ Lorentz spinor group, and $i, j$ the 'internal' $\overline{\mathrm{SO}(2,1)_{\text {int }}}$ indices.

Let the $\mathrm{SO}(2,1) N$-spinors $\varphi_{\alpha_{1} \ldots \alpha_{N}}$, symmetric in $\alpha_{1} \ldots \alpha_{N}$, be denoted by $\left(\frac{N}{2}, 0\right)$, and $\mathrm{SO}(2,1)_{\text {int }} L$-spinors by $\phi^{i_{1} \ldots i_{M}}$ by $\left(0, \frac{L}{2}\right)$. General $\operatorname{SO}(2,2)$ spinors $\left(\frac{N}{2}, \frac{L}{2}\right)=$ $\left(\frac{N}{2}, 0\right) \otimes\left(0, \frac{L}{2}\right)$ will then be denoted by $\psi_{\alpha_{1} \ldots \alpha_{N}}^{i_{1} \ldots i_{M}} ;$ spinors $\left(\frac{N}{2}, \frac{N}{2}\right)$ are then equivalent to $\mathrm{SO}(2,2) N$-tensors. In particular the basic $D=3, N=2$ spinors $\lambda_{\alpha}^{i}$ in our model describe the $\mathrm{SO}(2,2)$ vector

$$
\lambda_{A} \cong\left(\sigma_{A}\right)^{\alpha}{ }_{i} \lambda_{\alpha}^{i},
$$

where $A$ denotes the $\mathrm{SO}(2,2)$ four-vector indices and $\left(\sigma_{A}\right)^{\alpha}{ }_{i}$ are the $\mathrm{SO}(2,2) \sigma$-matrices analogous to the $\mathrm{SO}(3,1)$ matrices in (A.11). The extended spacetime coordinates $y_{a}^{\alpha \beta}$ (see (2.12)) and the variables $u_{\alpha \beta}^{a}$ (see (2.5)) describe second order $\operatorname{SO}(2,2)$ tensors $(1,1)$. Further one can show that the first three terms in (2.12) describe the $\mathrm{SO}(2,2)$ invariant contraction of two $\mathrm{SO}(2,2)$ tensors $(1,1)$, and the fourth term is the contraction of two $\mathrm{SO}(2,2)$ four-vectors. The mass-shell condition is defined by the $\mathrm{SO}(2,2)$-invariant scalar length of the $\mathrm{SO}(2,2)$ four-vector $\lambda_{A}$ (see (B.1)).

Our model (2.12) defines therefore the extension of the $N=2 D=3$ Shirafuji model to the vectorial model in $\mathrm{SO}(2,2)$ tensorial space $y_{a}^{\alpha \beta}$. Such a model cannot be however 
extended to a corresponding $O(3,3)$ twistorial model, because $\mathrm{SO}(3,3)$ twistors are described by the pair of primary $\mathrm{SO}(2,2)$ spinors $\left(\frac{1}{2}, 0\right) \oplus\left(0, \frac{1}{2}\right)$ which we denote as $\left(\lambda_{\alpha}, \lambda^{i}\right)$ and $\left(\omega_{\alpha}, \omega^{i}\right)$. The second pair of spinors should be defined in terms of $\mathrm{SO}(2,2)$ spacetime coordinates $x_{\alpha}^{i}$ by the $\mathrm{SO}(2,2)$ incidence relations

$$
\omega_{\alpha}=x_{\alpha}^{i} \lambda_{i}, \quad \omega^{i}=x_{\alpha}^{i} \lambda^{\alpha} .
$$

However, in this paper we did not use neither the simple spinors $\lambda_{\alpha}, \lambda^{i}$ nor the incidence relations (B.2) i.e., if we pass to an $\mathrm{SO}(2,2)$ interpretation of our model (2.12), we loose the corresponding $\mathrm{SO}(2,2)$ twistorial formulation.

Open Access. This article is distributed under the terms of the Creative Commons Attribution License (CC-BY 4.0), which permits any use, distribution and reproduction in any medium, provided the original author(s) and source are credited.

\section{References}

[1] C. Fronsdal, Massless particles, orthosymlectic symmetry and another Type of Kaluza-Klein theory in Mathematical Physics Studies. Vol. 8: Essays on Supersymmetry, Reidel, Dordrecht Netherlands (1986).

[2] I.A. Bandos and J. Lukierski, Tensorial central charges and new superparticle models with fundamental spinor coordinates, Mod. Phys. Lett. A 14 (1999) 1257 [hep-th/9811022] [INSPIRE].

[3] I.A. Bandos, J. Lukierski and D.P. Sorokin, Superparticle models with tensorial central charges, Phys. Rev. D 61 (2000) 045002 [hep-th/9904109] [INSPIRE].

[4] M.A. Vasiliev, Conformal higher spin symmetries of $4 d$ massless supermultiplets and osp $(L, 2 M)$ invariant equations in generalized (super)space, Phys. Rev. D 66 (2002) 066006 [hep-th/0106149].

[5] M. Plyushchay, D. Sorokin and M. Tsulaia, Higher spins from tensorial charges and $\operatorname{OSp}(N \mid 2 n)$ symmetry, JHEP 04 (2003) 013 [hep-th/0301067] [INSPIRE].

[6] S. Fedoruk and E. Ivanov, Master higher-spin particle, Class. Quant. Grav. 23 (2006) 5195 [hep-th/0604111] [INSPIRE].

[7] R. Penrose and M.A.H. MacCallum, Twistor theory: an approach to the quantization of fields and space-time, Phys. Rept. 6 (1972) 241 [InSPIRE].

[8] T. Shirafuji, Lagrangian mechanics of massless particles with spin, Prog. Theor. Phys. 70 (1983) 18 [InSPIRE].

[9] C. Fronsdal, Massless fields with integer spin, Phys. Rev. D 18 (1978) 3624 [InSPIRE].

[10] D. Sorokin, Introduction to the classical theory of higher spins, AIP Conf. Proc. 767 (2005) 172 [hep-th/0405069] [INSPIRE].

[11] A. Jevicki, K. Jin and Q. Ye, Collective dipole model of AdS/CFT and higher spin gravity, J. Phys. A 44 (2011) 465402 [arXiv:1106.3983] [InSPIRE].

[12] R. de Mello Koch, A. Jevicki, J.P. Rodrigues and J. Yoon, Holography as a gauge phenomenon in higher spin duality, JHEP 01 (2015) 055 [arXiv: 1408.1255] [INSPIRE]. 
[13] A.K.H. Bengtsson, I. Bengtsson, M. Cederwall and N. Linden, Particles, superparticles and twistors, Phys. Rev. D 36 (1987) 1766 [inSPIRE].

[14] I. Bengtsson and M. Cederwall, Particles, twistors and the division algebras, Nucl. Phys. B 302 (1988) 81 [INSPIRE].

[15] J. Tits, Lecture Notes in Mathematics. Vol. 40: Tabellen zu den einfachen Lie-Gruppen und ihren Darstellungen, Springer-Verlag, Berlin Germany (1967).

[16] A. Ferber, Supertwistors and conformal supersymmetry, Nucl. Phys. B 132 (1978) 55 [INSPIRE].

[17] Y. Eisenberg, Supertwistors and SuperPoincaré invariant actions for all linearized extended supersymmetric theories in four-dimensions, Mod. Phys. Lett. A 4 (1989) 195 [INSPIRE].

[18] Y. Eisenberg and S. Solomon, (Super)field theories from (super)twistors, Phys. Lett. B 220 (1989) 562.

[19] M.A. Vasiliev, Consistent equations for interacting massless fields of all spins in the first order in curvatures, Annals Phys. 190 (1989) 59 [INSPIRE].

[20] M.A. Vasiliev, Algebraic aspects of the higher spin problem, Phys. Lett. B 257 (1991) 111 [INSPIRE].

[21] M.A. Vasiliev, More on equations of motion for interacting massless fields of all spins in (3+1)-dimensions, Phys. Lett. B 285 (1992) 225.

[22] M.A. Vasiliev, Relativity, causality, locality, quantization and duality in the $S p(2 M)$ invariant generalized space-time, in Multiple facets of quantization and supersymmetry, Michael Marinov Memorial Volume, M. Olshanetsky and A. Vainshtein eds., World Scientific, Singapore (2002) [hep-th/0111119] [INSPIRE].

[23] O.A. Gelfond and M.A. Vasiliev, Higher rank conformal fields in the Sp(2M) symmetric generalized space-time, Theor. Math. Phys. 145 (2005) 1400 [hep-th/0304020] [INSPIRE].

[24] O.A. Gelfond, E.D. Skvortsov and M.A. Vasiliev, Higher spin conformal currents in Minkowski space, Theor. Math. Phys. 154 (2008) 294 [hep-th/0601106] [INSPIRE].

[25] O.A. Gelfond and M.A. Vasiliev, Unfolded equations for current interactions of $4 d$ massless fields as a free system in mixed dimensions, arXiv:1012.3143 [INSPIRE].

[26] M.A. Vasiliev, Holography, unfolding and higher-spin theory, J. Phys. A 46 (2013) 214013 [arXiv: 1203.5554] [INSPIRE].

[27] M.A. Vasiliev, Unfolded representation for relativistic equations in $(2+1)$ anti-de Sitter space, Class. Quant. Grav. 11 (1994) 649 [INSPIRE].

[28] A.K.H. Bengtsson, I. Bengtsson and L. Brink, Cubic interaction terms for arbitrary spin, Nucl. Phys. B 227 (1983) 31 [inSPIRE].

[29] F.A. Berends, G.J.H. Burgers and H. van Dam, On the theoretical problems in constructing interactions involving higher spin massless particles, Nucl. Phys. B 260 (1985) 295 [INSPIRE].

[30] V.E. Didenko and E.D. Skvortsov, Elements of Vasiliev theory, arXiv:1401.2975 [INSPIRE].

[31] M.A. Vasiliev, Higher-spin theory and space-time metamorphoses, Lect. Notes Phys. 892 (2015) 227 [arXiv:1404.1948] [INSPIRE]. 
[32] A.V. Barabanshchikov, S.F. Prokushkin and M.A. Vasiliev, Free equations for massive matter fields in $(2+1)$-dimensional anti-de Sitter space from deformed oscillator algebra, Theor. Math. Phys. 110 (1997) 295 [Teor. Mat. Fiz. 110N3 (1997) 372] [hep-th/9609034] [INSPIRE].

[33] S.F. Prokushkin and M.A. Vasiliev, Higher spin gauge interactions for massive matter fields in 3 -D AdS space-time, Nucl. Phys. B 545 (1999) 385 [hep-th/9806236] [InSPIRE].

[34] O.V. Shaynkman and M.A. Vasiliev, Scalar field in any dimension from the higher spin gauge theory perspective, Theor. Math. Phys. 123 (2000) 683 [hep-th/0003123] [InSPIRE].

[35] Y. Zinoviev, Frame-like gauge invariant formulation for massive high spin particles, Nucl. Phys. B 808 (2009) 185 [arXiv:0808.1778] [INSPIRE].

[36] N. Boulanger, C. Iazeolla and P. Sundell, Unfolding mixed-symmetry fields in AdS and the BMV conjecture: I. General formalism, JHEP 07 (2009) 013 [arXiv:0812.3615] [INSPIRE].

[37] D.S. Ponomarev and M.A. Vasiliev, Frame-like action and unfolded formulation for massive higher-spin fields, Nucl. Phys. B 839 (2010) 466 [arXiv: 1001.0062] [INSPIRE].

[38] N. Boulanger, D. Ponomarev, E. Sezgin and P. Sundell, New unfolded higher spin systems in $A d S_{3}$, arXiv: 1412.8209 [INSPIRE].

[39] S. Fedoruk and V.G. Zima, Bitwistor formulation of massive spinning particle, J. Kharkov Univ. 585 (2003) 39 [hep-th/0308154] [INSPIRE].

[40] S. Fedoruk and V.G. Zima, Bitwistor formulation of the spinning particle, in the Proceedings of the Supersymmetry and Quantum Symmetries International Workshop, Dubna, Russian Federation, 24-29 July, 2003 [hep-th/0401064].

[41] S. Fedoruk, A. Frydryszak, J. Lukierski and C. Miquel-Espanya, Extension of the Shirafuji model for massive particles with spin, Int. J. Mod. Phys. A 21 (2006) 4137 [hep-th/0510266] [INSPIRE].

[42] J.A. de Azcárraga, J.M. Izquierdo and J. Lukierski, Supertwistors, massive superparticles and к-symmetry, JHEP 01 (2009) 041 [arXiv:0808.2155] [INSPIRE].

[43] L. Mezincescu, A.J. Routh and P.K. Townsend, Supertwistors and massive particles, Annals Phys. 346 (2014) 66 [arXiv:1312.2768] [INSPIRE].

[44] K.P. Tod, Some symplectic forms arising in twistor theory, Rept. Math. Phys. 11 (1977) 339 [inSPIRE].

[45] Z. Perjés, Twistor variables of relativistic mechanics, Phys. Rev. D 11 (1975) 2031 [INSPIRE].

[46] Z. Perjés, Unitary space of particle internal states, Phys. Rev. D 20 (1979) 1857.

[47] L.P. Hughston, Lecture Notes In Physics. Vol. 97: Twistors and particles, Berlin Germany (1979).

[48] A. Bette, On a point-like relativistic and spinning particle, J. Math. Phys. 25 (1984) 2456.

[49] A. Bette, Directly interacting massless particles: A Twistor approach, J. Math. Phys. 37 (1996) 1724 [hep-th/9601017] [INSPIRE].

[50] A. Bette, J.A. de Azcárraga, J. Lukierski and C. Miquel-Espanya, Massive relativistic particle model with spin and electric charge from two twistor dynamics, Phys. Lett. B 595 (2004) 491 [hep-th/0405166] [INSPIRE]. 
[51] J.A. de Azcárraga, A. Frydryszak, J. Lukierski and C. Miquel-Espanya, Massive relativistic particle model with spin from free two-twistor dynamics and its quantization,

Phys. Rev. D 73 (2006) 105011 [hep-th/0510161] [INSPIRE].

[52] S. Fedoruk and J. Lukierski, Massive twistor particle with spin generated by Souriau-Wess-Zumino term and its quantization, Phys. Lett. B 733 (2014) 309 [arXiv: 1403.4127] [INSPIRE].

[53] L.C. Biedenharn, H.W. Braden, P. Truini and H. van Dam, Relativistic wavefunctions on spinor spaces, J. Phys. A 21 (1988) 3593.

[54] E. Sokatchev, Light cone harmonic superspace and its applications, Phys. Lett. B 169 (1986) 209.

[55] E. Sokatchev, Harmonic superparticle, Class. Quant. Grav. 4 (1987) 237 [INSPIRE].

[56] F. Delduc, S. Kalitsyn and E. Sokatchev, Learning the ABC of light cone harmonic space, Class. Quant. Grav. 6 (1989) 1561 [INSPIRE].

[57] R. Jackiw and V.P. Nair, Relativistic wave equations for anyons, Phys. Rev. D 43 (1991) 1933 [inSPIRE].

[58] R. Jackiw, Higher symmetries in lower dimensional models, NATO ASI C 409 (1993) 289.

[59] D.P. Sorokin and D.V. Volkov, (Anti)commuting spinors and supersymmetric dynamics of semions, Nucl. Phys. B 409 (1993) 547 [InSPIRE].

[60] J.L. Cortés and M.S. Plyushchay, Anyons: Minimal and extended formulations, Mod. Phys. Lett. A 10 (1995) 409 [hep-th/9405181] [InSPIRE].

[61] J.L. Cortés and M.S. Plyushchay, Anyons as spinning particles, Int. J. Mod. Phys. A 11 (1996) 3331 [hep-th/9505117].

[62] N.J. Vilenkin, Translations of Mathematical Monographs. Vol. 22: Special functions and the theory of group representations, American Mathematical Society, Providence U.S.A. (1968).

[63] V. Bargmann, Irreducible unitary representations of the Lorentz group, Ann. Math. 48 (1947) 568.

[64] W. Rühl, The Lorentz group and harmonic analysis, W.A. Benjamin, San Francisco U.S.A. (1970), pg. 299.

[65] I.A. Bandos, Superparticle in Lorentz harmonic superspace (in Russian), Sov. J. Nucl. Phys. 51 (1990) 906 [INSPIRE].

[66] F. Delduc, A. Galperin and E. Sokatchev, Lorentz harmonic (super)fields and (super)particles, Nucl. Phys. B 368 (1992) 143 [INSPIRE].

[67] S. Fedoruk and V.G. Zima, Covariant quantization of $d=4$ Brink-Schwarz superparticle with Lorentz harmonics, Theor. Math. Phys. 102 (1995) 305 [hep-th/9409117] [INSPIRE].

[68] A.P. Balachandran, G. Marmo, B.S. Skagerstam and A. Stern, Gauge symmetries and fiber bundles: applications to particle dynamics, Lect. Notes Phys. 188 (1983) 1.

[69] L. Schulman, A Path integral for spin, Phys. Rev. 176 (1968) 1558 [INSPIRE].

[70] C.A. Orzalesi, Charges and generators of symmetry transformations in quantum field theory, Rev. Mod. Phys. 42 (1970) 381 [InSPIRE].

[71] A.S. Galperin, E.A. Ivanov, V.I. Ogievetsky and E.S. Sokatchev, Harmonic Superspace, Cambridge University Press, Cambridge U.K. (2001). 
[72] I.M. Gelfand, M.I. Graev and N.J. Vilenkin, Generalized functions: fntegral geometry and representation theory, Academic Press, New York U.S.A. (1966).

[73] E.-M. Corson, Introduction to tensors, spinors and relativistic wave equations, Blackie and Son, London U.K. (1957).

[74] S. Weinberg, Feynman rules for any spin, Phys. Rev. 133 (1964) B1318.

[75] J. Lukierski and A. Nowicki, Quaternionic six-dimensional (super)twistor formalism and composite (super)spaces, Mod. Phys. Lett. A 6 (1991) 189 [InSPIRE].

[76] Z. Hasiewicz, P. Morawiec and J. Lukierski, Quaternionic six-dimensional (super)twistor formalism and composite (super)spaces, Phys. Lett. B 130 (1983) 55 [INSPIRE].

[77] C. Duval and P.A. Horvathy, Chiral fermions as classical massless spinning particles, Phys. Rev. D 91 (2015) 045013 [arXiv: 1406.0718] [inSPIRE].

[78] J.A. de Azcárraga, J.P. Gauntlett, J.M. Izquierdo and P.K. Townsend, Topological extensions of the supersymmetry algebra for extended objects,

Phys. Rev. Lett. 63 (1989) 2443 [INSPIRE].

[79] C. Chryssomalakos, J.A. de Azcárraga, J.M. Izquierdo and J.C. Pérez Bueno, The geometry of branes and extended superspaces, Nucl. Phys. B 567 (2000) 293 [hep-th/9904137] [INSPIRE].

[80] J.A. de Azcárraga and J.M. Izquierdo, Some geometrical aspects of M-theory, Lisbon Conference on Geometry and Physics, Lisbon Portugal (2007), AIP Conf. Proc. 1023 (2008) 57 [INSPIRE].

[81] J.A. de Azcárraga and J. Lukierski, Supersymmetric Particles with Internal Symmetries and Central Charges, Phys. Lett. B 113 (1982) 170 [INSPIRE].

[82] J.A. de Azcárraga and J. Lukierski, Gupta-Bleuler quantization of massive superparticle models in $D=6, D=8$ and $D=10$, Phys. Rev. D 38 (1988) 509 [INSPIRE].

[83] E.S. Fradkin and M.A. Vasiliev, Cubic Interaction in Extended Theories of Massless Higher Spin Fields, Nucl. Phys. B 291 (1987) 141 [InSPIRE].

[84] H. van Dam and M.J.G. Veltman, Massive and massless Yang-Mills and gravitational fields, Nucl. Phys. B 22 (1970) 397 [InSPIRE].

[85] V.I. Zakharov, Linearized gravitation theory and the graviton mass, JETP Lett. 12 (1970) 312 [INSPIRE].

[86] M. Porrati, No van Dam-Veltman-Zakharov discontinuity in AdS space, Phys. Lett. B 498 (2001) 92 [hep-th/0011152] [InSPIRE].

[87] S. Deser and A. Waldron, (Dis)continuities of massless limits in spin $3 / 2$ mediated interactions and cosmological supergravity, Phys. Lett. B 501 (2001) 134 [hep-th/0012014] [INSPIRE].

[88] V.I. Ogievetsky and I.V. Polubarinov, Interacting field of spin 2 and the Einstein equations, Ann. Phys. (NY) 35 (1965) 167.

[89] E.C.G. Stueckelberg, Interaction energy in electrodynamics and in the field theory of nuclear forces, Helv. Phys. Acta 11 (1938) 225 [inSPIRE].

[90] J. Lukierski, Renormalizability of higher-spin theories, Nuovo Cim. 38 (1965) 1407. 
[91] H. Ruegg and M. Ruiz-Altaba, The Stueckelberg field, Int. J. Mod. Phys. A 19 (2004) 3265 [hep-th/0304245] [INSPIRE].

[92] R.R. Metsaev, Arbitrary spin conformal fields in (A)dS, Nucl. Phys. B 885 (2014) 734 [arXiv: 1404.3712] [INSPIRE].

[93] N. Beisert, M. Bianchi, J.F. Morales and H. Samtleben, Higher spin symmetry and $N=4$ SYM, JHEP 07 (2004) 058 [hep-th/0405057] [INSPIRE].

[94] M. Bianchi, P.J. Heslop and F. Riccioni, More on La Grande Bouffe, JHEP 08 (2005) 088 [hep-th/0504156] [INSPIRE].

[95] M.A. Vasiliev, Multiparticle extension of the higher-spin algebra, Class. Quant. Grav. 30 (2013) 104006 [arXiv:1212.6071] [INSPIRE].

[96] O.A. Gelfond and M.A. Vasiliev, Operator algebra of free conformal currents via twistors, Nucl. Phys. B 876 (2013) 871 [arXiv:1301.3123] [INSPIRE].

[97] M.A. Vasiliev, Extended Higher Spin Superalgebras and Their Realizations in Terms of Quantum Operators, Fortsch. Phys. 36 (1988) 33 [INSPIRE].

[98] S.E. Konstein and M.A. Vasiliev, Extended Higher Spin Superalgebras and Their Massless Representations, Nucl. Phys. B 331 (1990) 475 [inSPIRE].

[99] N. Boulanger, D. Ponomarev, E.D. Skvortsov and M. Taronna, On the uniqueness of higher-spin symmetries in AdS and CFT, Int. J. Mod. Phys. A 28 (2013) 1350162 [arXiv:1305.5180] [INSPIRE].

[100] T.D. Lee, S. Weinberg and B. Zumino, Algebra of Fields, Phys. Rev. Lett. 18 (1967) 1029 [INSPIRE].

[101] J.J. Sakurai, Currents and Mesons, University of Chicago Press, Chicago U.S.A. (1969).

[102] M. Flato and C. Fronsdal, One Massless Particle Equals Two Dirac Singletons: Elementary Particles in a Curved Space. 6., Lett. Math. Phys. 2 (1978) 421 [InSPIRE].

[103] J.-M. Souriau, Structure des systèmes dynamiques, Dunod, Paris France (1970) [Progress in Mathematics. Vol. 149: Structure of dynamical systems: a symplectic view of physics, Boston U.S.A. (1997)].

[104] H.P. Künzle, Canonical dynamics of spinning particles in gravitational and electromagnetic fields, J. Math. Phys. 13 (1972) 739 [InSPIRE].

[105] A. Pashnev and M. Tsulaia, Description of the higher massless irreducible integer spins in the BRST approach, Mod. Phys. Lett. A 13 (1998) 1853 [hep-th/9803207] [INSPIRE].

[106] C. Burdik, A. Pashnev and M. Tsulaia, On the Mixed symmetry irreducible representations of the Poincaré group in the BRST approach, Mod. Phys. Lett. A 16 (2001) 731 [hep-th/0101201] [INSPIRE].

[107] I.L. Buchbinder, A. Pashnev and M. Tsulaia, Lagrangian formulation of the massless higher integer spin fields in the AdS background, Phys. Lett. B 523 (2001) 338 [hep-th/0109067] [INSPIRE].

[108] I.L. Buchbinder, V.A. Krykhtin and M. Tsulaia, Lagrangian formulation of massive fermionic higher spin fields on a constant electromagnetic background, arXiv:1501.03278 [INSPIRE]. 